\title{
MODELS IN THE DESIGN AND VALIDATION OF EDDY CURRENT INSPECTION FOR CRACKING IN THE SHUTTLE REACTION CONTROL SYSTEM THRUSTER
}

\author{
John C. Aldrin ${ }^{1}$, Phillip A. Williams ${ }^{2}$, Buzz Wincheski ${ }^{2}$ \\ ${ }^{1}$ Computational Tools, Gurnee, IL 60031, USA \\ ${ }^{2}$ NASA Langley Research Center, Hampton, VA 23681, USA
}

\begin{abstract}
A case study is presented for using models in eddy current NDE design for crack detection in Shuttle Reaction Control System thruster components. Numerical methods were used to address the complex geometry of the part and perform parametric studies of potential transducer designs. Simulations were found to show agreement with experimental results. Accurate representation of the coherent noise associated with the measurement and part geometry was found to be critical to properly evaluate the best probe designs.
\end{abstract}

Keywords: eddy current, cracks, finite element method, model-assisted POD, volume element method PACS: 81.70.Ex

\section{INTRODUCTION}

During the life of the shuttle program, cracking in the relief radius of the primary reaction control system thruster has been identified [1]. This problem is shown in Figure 1(a) where intergranular cracking extending from the relief radius propagates toward the acoustic cavity. Eddy current techniques have recently been developed to detect cracking in Shuttle Reaction Control System (RCS) thruster components [2-3]. An orthogonal dual coil probe shown in Figure 1(b) was developed to detect such cracks [2]. In addition, remote field eddy current designs have been explored to find deeply buried flaws [3]. Recently, the orthogonal probe has been successfully validated for detecting larger cracks through a probability of detection study [4].

Although an adequate design was developed and validated, there is an interest in better leveraging models to improve the design and validation of NDE techniques in the future. In general, modeling and simulation can produce significant benefits at several stages of the NDT technique development process [5]. First, models can be used to build a fundamental understanding of the inspection problem and aid in the interpretation of raw measurement data. Also, the design of eddy current probes can greatly benefit from parametric studies using accurate measurement models. During the validation process, model-assisted probability of detection (MAPOD) procedures have the potential to evaluate the reliability of an NDT technique at a lower cost with respect to conventional POD studies [5-6]. Some simulated studies for the RCS inspection were performed exploring changes in field strength with depth for different probe configurations [7]; however, more accurate measurement models are needed to better explore design and validation scenarios.

A case study on the thruster inspection using eddy current NDE is presented for the use of models in the design and validation of NDE techniques. A primary objective of this 
paper is to develop a measurement model that closely represents the NDE inspection response. Two numerical methods, the finite element method and the volume integral method, are used to simulate the complex geometry of the part and results are compared with prior experimental work. Sensitivity studies are presented to determine the ideal probe orientations for varying crack lengths and explore the best NDE technique design. Lastly, the concept of a full model-assisted POD evaluation is discussed and applied for properly comparing different NDE technique designs.

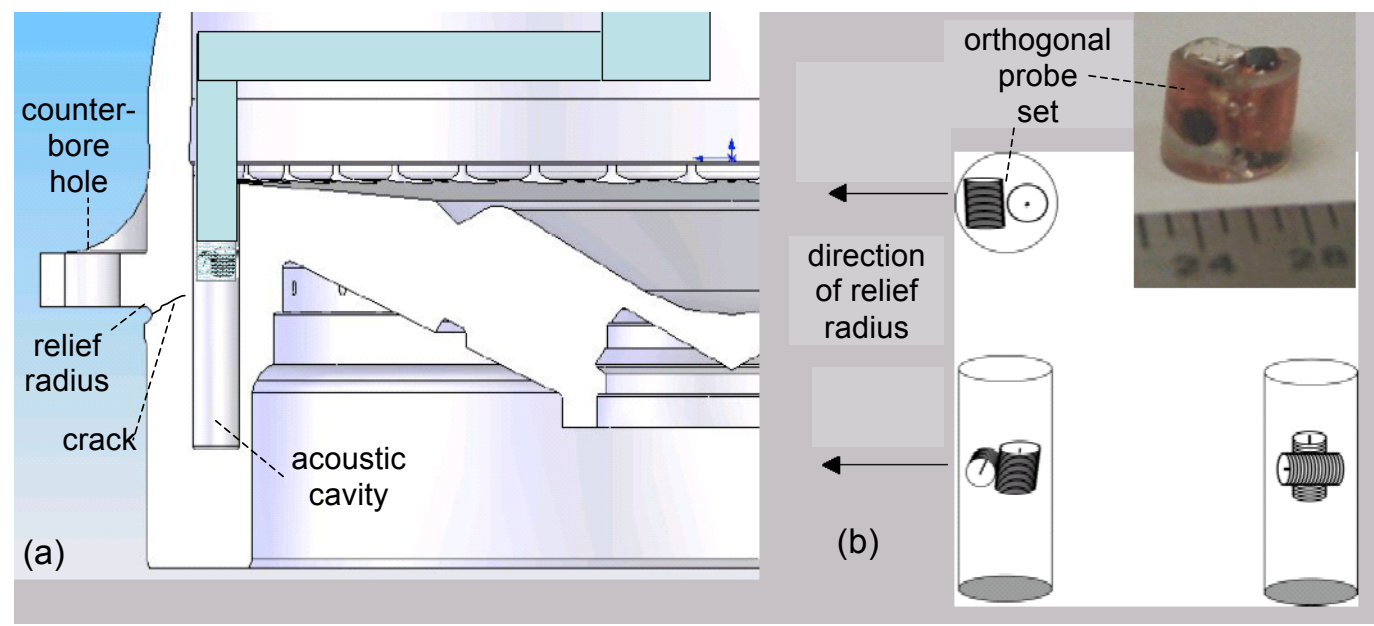

FIGURE 1. Diagram of (a) thruster cross-section with hole and relief radius and (b) orthogonal eddy current probe set [2].

\section{MODELING APPROACH}

Before selecting the appropriate modeling approach for this case study, all of the critical factors in the NDE measurement problem, including part geometry, part material properties, crack characteristics and NDE technique design, must be assessed in terms of their importance on the eddy current response. For the RCS thruster inspection, important factors concerning the eddy current probe design include the use of one or more coils (secondary coils as reference coils), coil orientations (three different axial directions with respect to the cavity), coil dimension, and probe frequency $(1-50 \mathrm{kHz})$. Details of the inspection model are as follows. The thruster material is a niobium alloy with a conductivity of $13.2 \%$ IACS [8]. The intergranular cracks were modeled as open cracks. Crack orientation from the relief radius varies between $30^{\circ}-60^{\circ}$. Another critical point concerns the degree to which the geometry can be simplified and its role on simulation time. From a life prediction perspective, projected crack depth (remaining wall thickness for example $0.020^{\prime \prime}$ to $0.060^{\prime \prime}$ ) is more critical to measure than actual crack depth. All response plots are generated in terms of remaining material thickness. The frequency of all of the results in this study is $12 \mathrm{kHz}$.

To accurately simulate an eddy current measurement for a variety of complex probe designs and test sample configurations, numerical methods, such as the finite difference method, finite element method (FEM), boundary integral equation method, the volume integral method, and meshless methods, have been developed [5]. Due to their ability to simulate complex problems efficiently in finite domains, the finite element method and volume integral method are used in this study. The numerical formulation of the finite element method solves the governing equations for each element using either Galerkin's method or a variation approach [9]. The resulting system of equations for the meshed domain is then solved most often in terms of magnetic vector potential. The measurement 
model for an eddy current coil can then be expressed in terms of changes in resistance and inductance, associated with dissipated power $(\mathrm{P})$ in the region of the conductor and the stored energy $(\mathrm{W})$ in the whole solution domain $(\Gamma)$, respectively

$$
Z=R+j \omega L=\frac{P}{I^{2}}+\frac{2 j \omega W}{I^{2}} .
$$

Using the finite element solution for the electric and magnetic field intensities in the solution domain, the dissipated energy in the conductor $\left(\Gamma^{\prime}\right)$ and stored energy in the whole solution domain $(\Gamma)$ can be calculated as follows:

$$
\begin{gathered}
P=\int J^{e} E^{*} d \Gamma^{\prime}, \\
W=\frac{1}{2} \int H B^{*} d \Gamma .
\end{gathered}
$$

To evaluate the change in impedance in a conductor due solely to the presence of a crack or notch, the difference in impedance must be calculated from two separate problems representing 1) a pristine sample and 2) a sample containing a notch. Advantages of the FEM model include the fact that the solution is valid and available in the entire domain, and the ability to address problems with awkward geometries, inhomogeneous media and nonlinear materials. The primary disadvantage is simulation time for large problems.

An alternative method to direct FEM solution concerns the use of an approximate representation of the thruster problem using the volume integral method. The volume integral equation or volume integral method models anomalous regions as a fictitious current source, which is directly related to departures in electrical conductivity and magnetic permeability from the host material. Green's functions for infinitesimal current sources in multi-layered materials are applied and the method of moments (MoM) is used to solve the volume integral equation. The advantage of this formulation is that only the region of the scatterer needs to be discretized. Because of the regular grid, the resulting matrix has an advantageous structure where very large problems can be solved efficiently using the conjugate-gradient method. $\mathrm{VIC}-3 \mathrm{D}^{\circledR}$ is an implementation of the volume integral method where the geometry of a part can be built using a series of material layers (in Cartesian or cylindrical coordinates) with primitive geometries to model the 'flaw' or 'anomalous' region across the multiple layers [10]. VIC-3D ${ }^{\circledR}$, which is catered to eddy current simulation, also has the ability to quickly simulate numerous probe configurations of interest including varying orientation.

The initial modeling approach for this problem was to use the finite element method and the volume integral method to separately address the complex geometry of the part and perform parametric studies of potential transducer designs, respectively. When using the finite element method, it is prudent to limit the domain of the mesh only to the portion of the part geometry that impacts the eddy current measurement. Figure 2(a) represents an initial level of simplification from the complete thruster geometry to minimize computational expense. Figures 2(b) and 2(c) show the model implementation in the Opera3 ${ }^{\circledR}$, the electromagnetic FEM solver. An open notch at an angle of $45^{\circ}$ was used to represent the crack in the model. Only a single coil with varying position in the acoustic cavity was simulated. For the volume integral method in VIC-3D ${ }^{\circledR}$, additional approximations to the model were considered in order to improve simulation time. First, to study the effect of probe position, probe design (shape, orientation) and operating frequency, and varying crack length requiring numerous simulations, the thruster geometry 

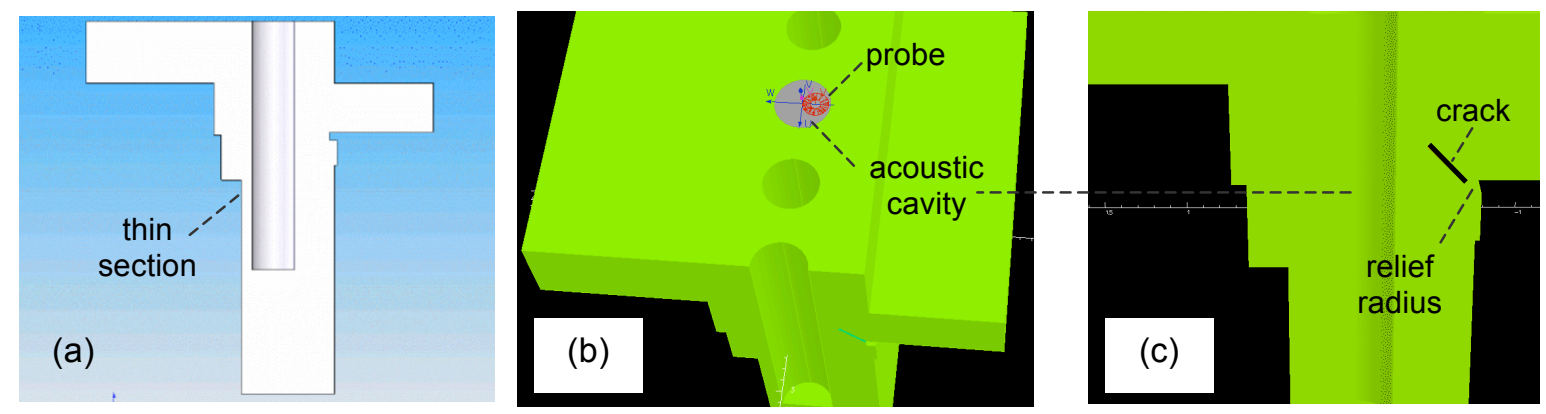

FIGURE 2. Diagram of (a) simplified geometry for thruster model with (b) and (c) views from Opera3 $\mathrm{D}^{\circledR}$.
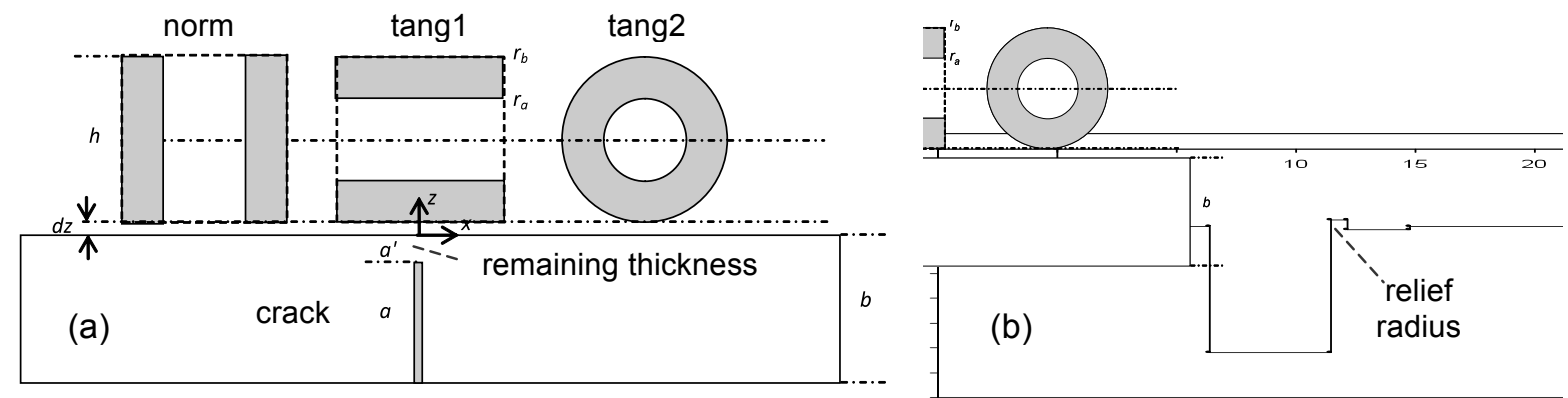

FIGURE 3. (a) Approximate model (1) of inspection problem in VIC-3D ${ }^{\circledR}$ for three different probe orientations: normal (norm), tangential (tang1) and tangential (tang2) and (b) approximate 2D representation of the thruster geometry profile (only side with relief radius displayed).

was locally approximated to be an infinite flat plate as shown in Figure 3(a). Although VIC-3D ${ }^{\circledR}$ could not easily represent the full 3D model as in the FEM model, an approximate 2D representation of the geometry as shown in Figure 3(b) was also solved to understand the interaction effect of probe orientation and part geometry, including the crack condition.

\section{MODELING RESULTS}

Figure 4(a) displays the FEM results for the eddy current probe response in terms of dissipated power (P) and the stored energy (W). Although the trends observed in the response are correlated with the part geometry, given how the field generated by the small coil was solved using FEM with a finite irregular tetrahedral mesh, there is irregularity in the solution. The challenge here is that increasing the mesh density to get a smoother absolute response will significantly increase solution time. Figure 4(b) displays differential results generated by solving both the 'no flaw' and 'with flaw' conditions and taking the difference. Mesh error is considerably minimized when using a differential approach; however, simulation time (for 52 runs at $2 * 26$ probe locations) is still on the order of 36 hours. Even though some time could be saved by using an analytical model for the field generated by the coil in the part and working to reduce mesh size, an alternative is needed.

Experimental results are presented in Figures 5(a) and 5(b) for the vertical response of the eddy current measurement for (a) no crack and (b) with crack cases. The two curves in each figure represent the scan in and out of the acoustic cavity. There is a characteristic response for the 'no crack' with a sharp change as the cavity is entered (on the left) and then a slight rise as the probe passes the thin section deeper in the cavity (to the right). (Note, the plot does not include the response when the probe reaches the very end of the cavity.) All experimental results are acquired with the orthogonal probe set. This corresponds with the 'tang2' coil orientation being on the side near the relief radius and 
crack. For more information on the experimental measurements, see references [2,4,7]. The crack results shown in Figure 5(b) highlight an additional characteristic Gaussian response as the crack region (with $0.020^{\prime \prime}$ remaining thickness) is scanned. Simulated results are provided by VIC-3D ${ }^{\circledR}$ to represent this test configuration. Instead of a complete orthogonal probe model, the results are only presented for the tang2 coil orientation. In general, the characteristic response for the part and crack condition as found in the experimental data are well represented in the simulated results shown in Figure 5(c).


FIGURE 4. Plots of (a) absolute FEM solution for eddy current response from an unflawed part and (b) differential FEM solution for eddy current response for a crack (with respect to unflawed specimen).
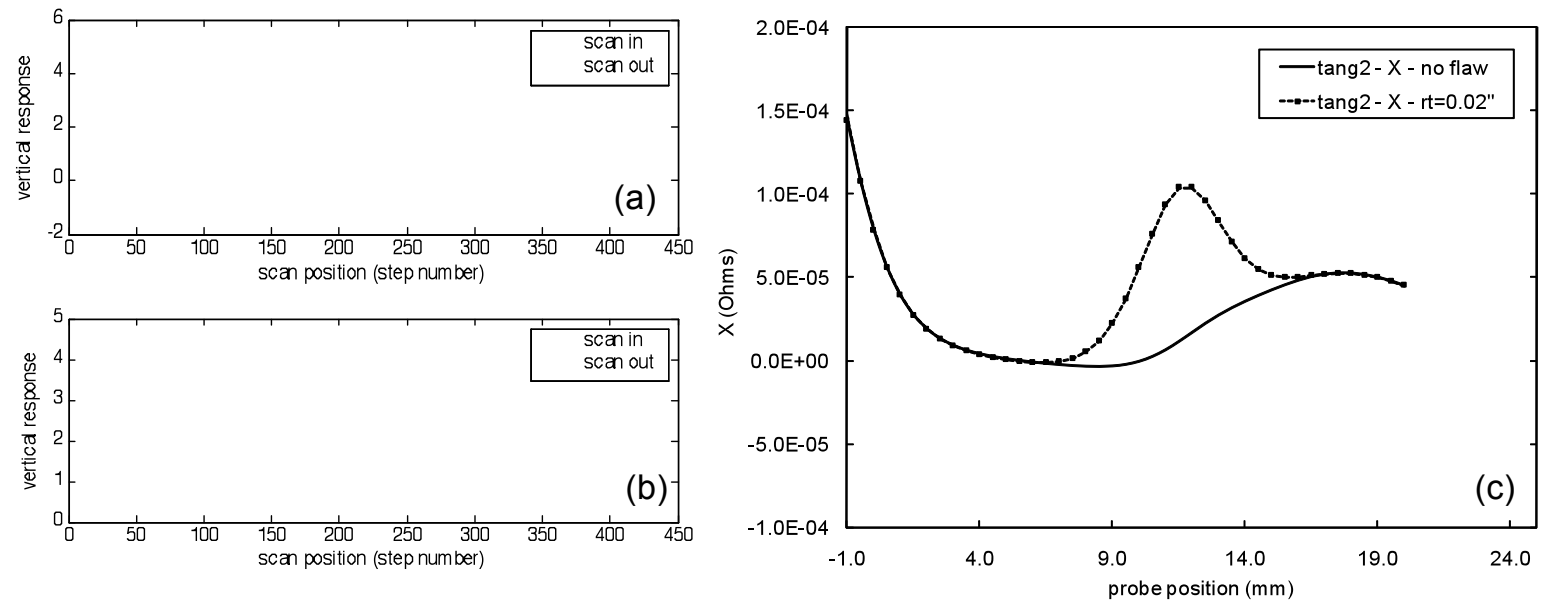

FIGURE 5. Plots of experimental results for vertical component for (a) no crack and (b) with crack cases, and (c) simulated (VIC-3D ${ }^{\circledR}$ ) results for no crack and with crack $\left(0.020^{\prime \prime}\right.$ remaining thickness).

Figures 6(a) and 6(b) present plots of the crack measurement (peak of the localized Gaussian) response with respect to varying remaining thickness for experimental [4] and simulated VIC-3D ${ }^{\circledR}$ results respectively. Note, the experimental data for the $0.020^{\prime \prime}$ remaining thickness are used to calibrate the model with the experimental data. When comparing the results for the 0.040" and 0.060" remaining thickness cases, the trend in the simulated results is found to be similar but somewhat lower than the experimental results. This error is likely due to the simulation using only the 'tang2' coil orientation and not representing the full orthogonal probe set.

Figures 7(a) and 7(b) display results for the eddy current measurement for a change in resistance and reactance for varying probe orientation and remaining thickness. In general, the normal ('norm') probe orientation was found to have the greatest sensitivity to cracks, with the 'tang2' orientation being about half as sensitive, and the 'tang1' orientation far 

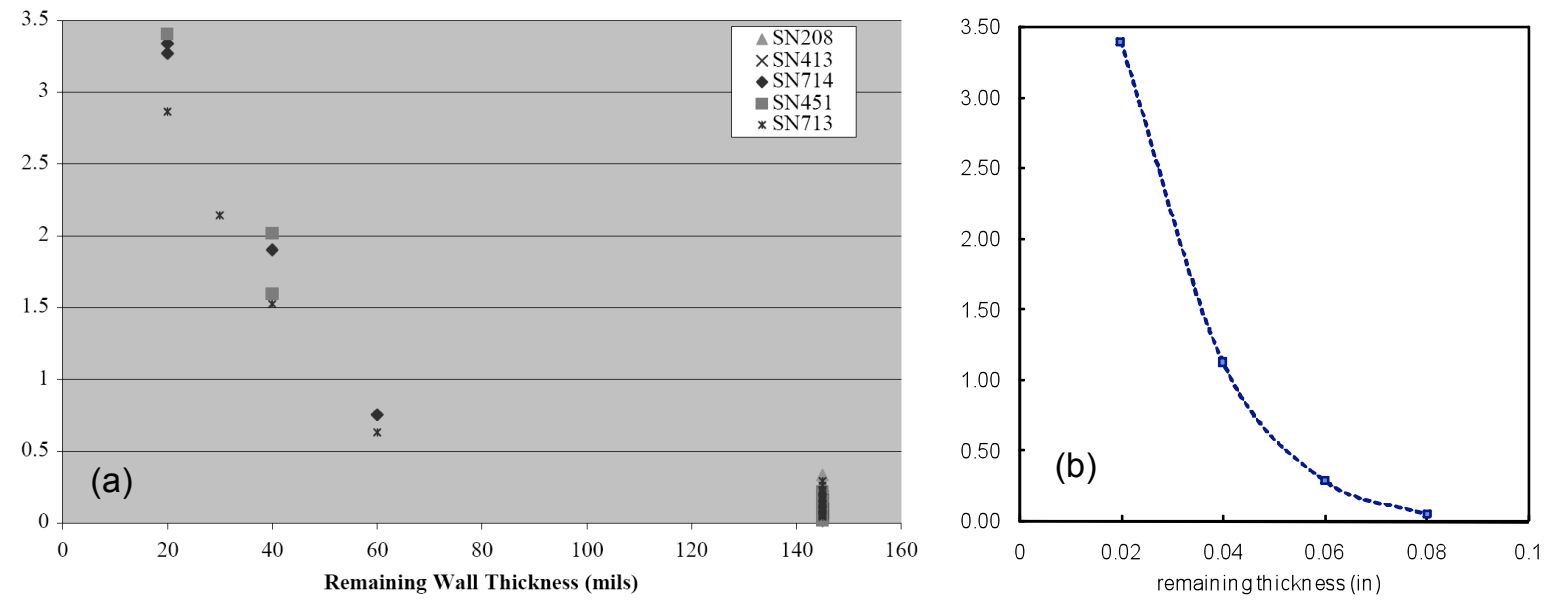

FIGURE 6. Plots of crack measurement response for varying remaining wall thickness: (a) experimental results $\lceil 4\rceil$ and (b) simulated VIC-3D ${ }^{\circledR}$ results (normalized to 0.020 " value for experimental data).
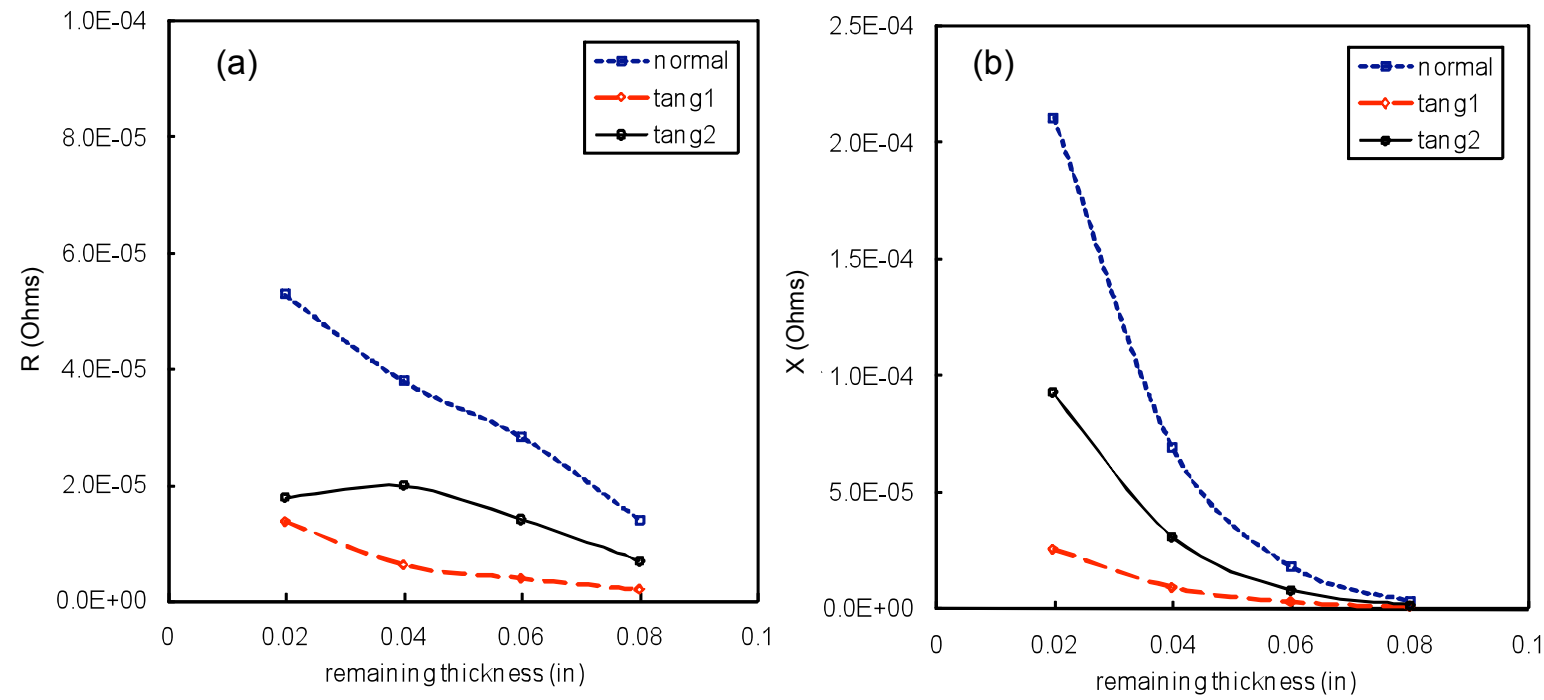

FIGURE 7. Plots of crack response in terms of the (a) resistance and (a) reactance of an eddy current measurement for varying probe orientation and remaining thickness.

lower. Prior work has shown when simply looking at the field generated by the normal and tangential (tang2) orientations, the tangential coil had advantages with deeper field penetration [7]. This is clearly true, but when considering the detection problem, the normal coil is more sensitive at all depths with respect to a coil orientated in a tangential direction. However, that does not mean that the normal coil is optimal. Not only must signal strength be considered for comparing different probe orientations and crack depths, but the noise condition for each of these probes must be completely evaluated. For example, the normal coil orientation has been shown experimentally to increase signal noise due to larger lift-off and probe wobble effects [7]. This concept to evaluate the signal and noise response for varying crack length is essentially the objective of model-assisted probably of detection evaluation and will be discussed in the next section.

\section{MODEL-ASSISTED POD EVALUATION IN DESIGN OPTIMIZATION}

To validate the performance of new inspection techniques, probability of detection (POD) studies can be performed. A model-assisted strategy for the design and execution of 
POD studies has been proposed to help mitigate the validation costs and to improve POD evaluation quality by addressing a wider array of inspection variables [6,5]. Using computer and empirical models to address variables that cannot be easily recreated in experimental samples is a significant opportunity. The full model assisted approach uses advanced computer simulations to model the inspection process and determines the POD for the inspection technique through a combination of experimental and simulated data. Recent work has demonstrated the potential of the full MAPOD methodology incorporating computer simulation for the inspection of cracks around fastener sites in a two layer aircraft structure inspection performed with an eddy current technique [5].

In addition to using a full model-assisted POD evaluation for validation purposes, to properly compare competing NDE technique designs, a MAPOD evaluation that incorporates an assessment of the signal and noise distributions as a function of varying crack length is quite necessary. To perform a model-assisted POD evaluation, all critical factors that impact the distributions for the noise and crack response must be evaluated. Concerning the noise distribution for this cases study, all variations due to the measurement method (use of a reference sensor or self-calibration approach), the part (for example variation in the location of the crack) and the probe (specifically orientation and liftoff from the surface) must be considered. In addition, factors that are critical for evaluating the response as a function of crack size include variation in the crack characteristics (in particular orientation) and the interaction of the probe with the unique part geometry.

Performing a full model-assisted POD evaluation is not a trivial task; however, assumptions can be made in the evaluation process that can lead to a reasonable answer without excessive computational expense. For this case study, the interaction between the probe orientation and part geometry will be considered. Figure 8(a) shows a diagram of the three probes orientations scanned over the part geometry broken into two sides with Figures 8(b) and 8(c) representing the simulated results (in VIC-3D ${ }^{\circledR}$ ) for the upper and lower sections, respectively. Although the normal coil is twice as sensitive to the cracks with respect to the tangential (2) coil, the normal coil was found to be an order of magnitude more sensitive to the part geometry. Thus, the coherent noise generated from the part, which also impacts sensitivity to probe wobble and liftoff, is not ideal for the normal coil design and indicates that the tangential (2) design is a better choice.
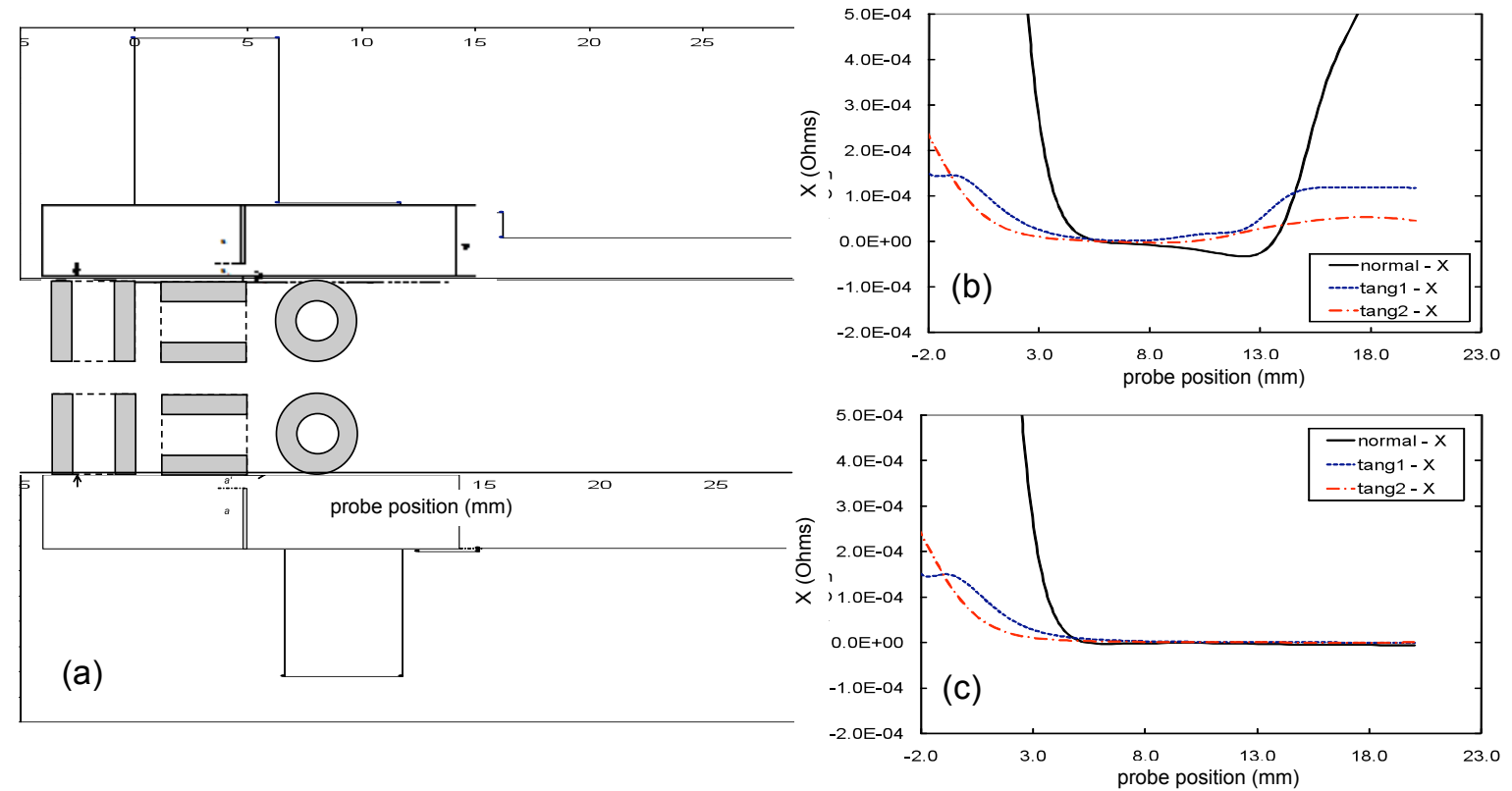

FIGURE 8. (a) Diagram of approximate model of inspection problem for three different probe orientations with simulated results (in VIC-3D ${ }^{\circledR}$ ) for the (b) upper and (c) lower sections respectively. 


\section{CONCLUSIONS AND RECOMMENDATIONS}

A case study is presented for using models in eddy current NDE design for crack detection in Shuttle Reaction Control System thruster components. Simulations using the volume integral method with some model approximations were found to show good agreement with experimental results. Sensitivity studies were performed to explore the ideal probe orientations for varying crack lengths. However, it was found that to properly compare competing NDE technique designs, a full model-assisted POD evaluation that incorporates an assessment of the signal and noise distributions as a function of varying flaw size (crack length) is really necessary. Although performing a full model-assisted POD evaluation is not a trivial task, assumptions can be made in the evaluation process that can lead to a reasonable answer without excessive computational expense. Future work is proposed to perform a more complete MAPOD study, explore hybrid models to efficiently solve for multiscale geometries, and investigate optimum designs for improving detection of deep cracks.

\section{ACKNOWLEDGEMENTS}

Support was provided by the NASA Super Problem Resolution Team (SPRT) including program support from Bill Prosser, Bill Winfree, Roy Savage, and ATK. Support for VIC$3 \mathrm{D}^{\circledR}$ was provided by Victor Technologies LLC.

\section{REFERENCES}

1. R. A. MacKay, S. W. Smith, S. R. Shah, and R. S. Piascik, "Reaction control system thruster cracking consultation: NASA Engineering and Safety Center (NESC) Materials Super Problem Resolution Team (SPRT) Findings, NASA/TP-2005-214053.

2. B. Wincheski, J. Simpson, and A. Koshti, "Development of Eddy Current Technique for the Detection of Stress Corrosion Cracking in Space Shuttle Primary Reaction Control Thrusters," NASA/TP-2007-214878, (June, 2007).

3. Y. Sun, C. Sun, H. Zhu, and B. Wincheski, "A Tool Measuring Remaining Thickness of Notched Acoustic Cavities in Primary Reaction Control Thruster NDI Standards", Review of Progress in QNDE, Presentation, (Portland OR, 2006).

4. B. Wincheski, "Validation Test Results for Orthogonal Probe Eddy Current Thruster Inspection System," NASA/TM-2007-215087, (November, 2007).

5. J.C. Aldrin, and J.S. Knopp, "Modeling and Simulation for Nondestructive Testing with Applications to Aerospace Structures", Materials Evaluation, Vol. 66, n 1, pp. 53-59, (2008).

6. R.B. Thompson, "Using Physical Models of the Testing Process in Determination of Probability of Detection," Materials Evaluation, Vol. 59, n 7, pp. 861-865 (2001).

7. B. Wincheski, P. Williams, and J. Simpson, "Analysis of Eddy Current Capabilities for the Detection of Outer Diameter Cracking In Small Bore Metallic Structures," Review of Progress in QNDE, Vol. 27, pp. 384-391, (2008).

8. Web site: http://www.rembar.com/niobium.htm.

9. D.C. Carpenter, "Use of the Finite Element Method in Simulation and Visualization of Electromagnetic Nondestructive Testing Applications," Materials Evaluation, Vol. 58, n 7, pp. 877-881, (2000).

10. R. Murphy, H. Sabbagh, A. Chan, and E. Sabbagh, "A Volume-integral Code for Electromagnetic Nondestructive Evaluation," Proceedings of the 13th Annual Review of Progress in Applied Computational Electromagnetics, Monterey, CA, (March 1997). 


\title{
Models in the Design and Validation of Eddy Current Inspection for Cracking in the Shuttle Reaction Control System Thruster
}

\author{
John C. Aldrin, \\ Computational Tools \\ Phillip A. Williams, Buzz Wincheski \\ NASA Langley Research Center \\ $35^{\text {th }}$ Annual Review of Progress in QNDE \\ July 23, 2008 - Chicago, Illinois
}




\section{Outline}

- Background - RCS Thruster

- Modeling Approach

- Results

- FEM (Opera-3D ${ }^{\circledR}$ )

- Volume Integral Method (VIC-3D ${ }^{\circledR}$ )

- Design Trends

- Model-assisted POD Evaluation

- Protocol

- Preliminary Results

- Summary and Future Work 


\section{Background - RCS Thruster Problem}

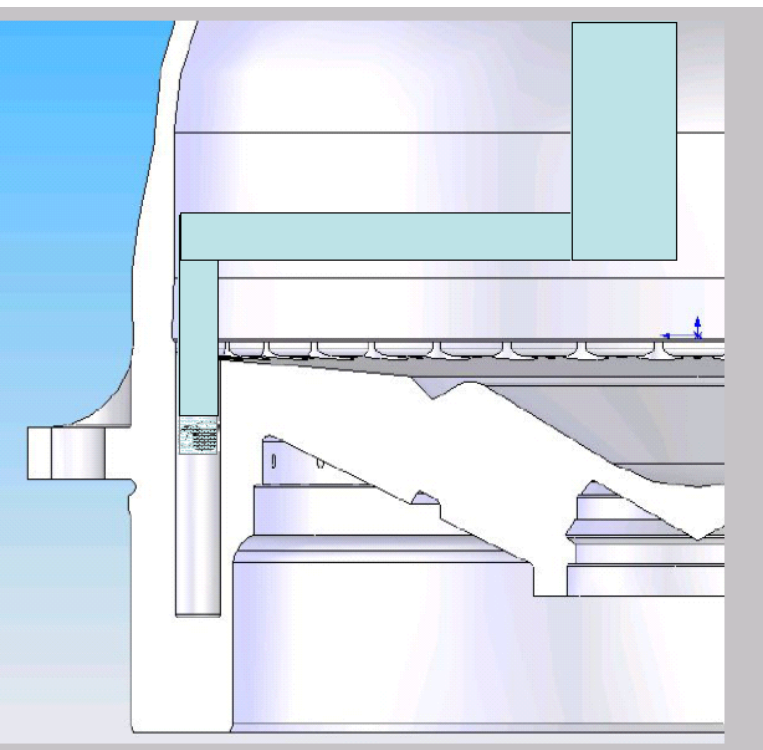

Thruster Cut-away

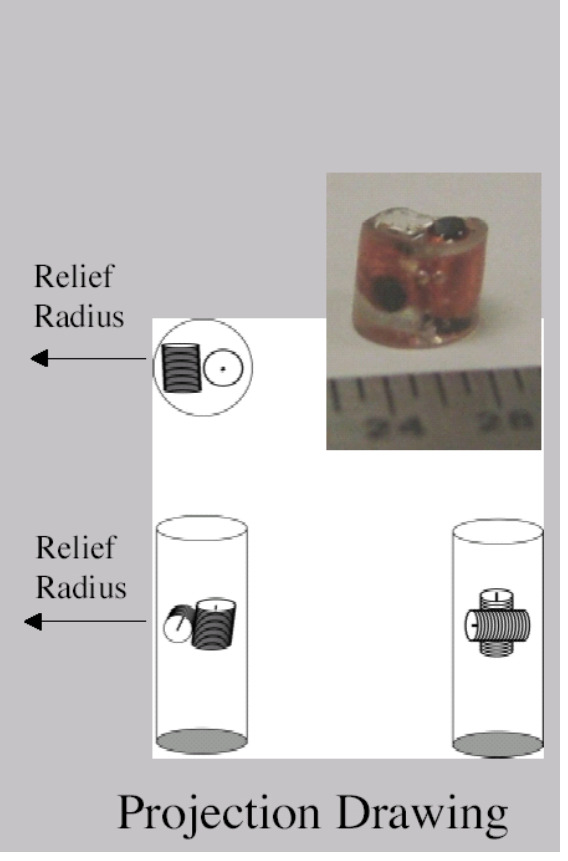

Projection Drawing



Observations for PRCS Thruster Inspection for Model:

- $\quad$ Cracks may emanate from relief radius or up flange. (initially model from relief radius.)

- $\quad$ Cracks are intergranular but generally open. (initially model as open cracks.)

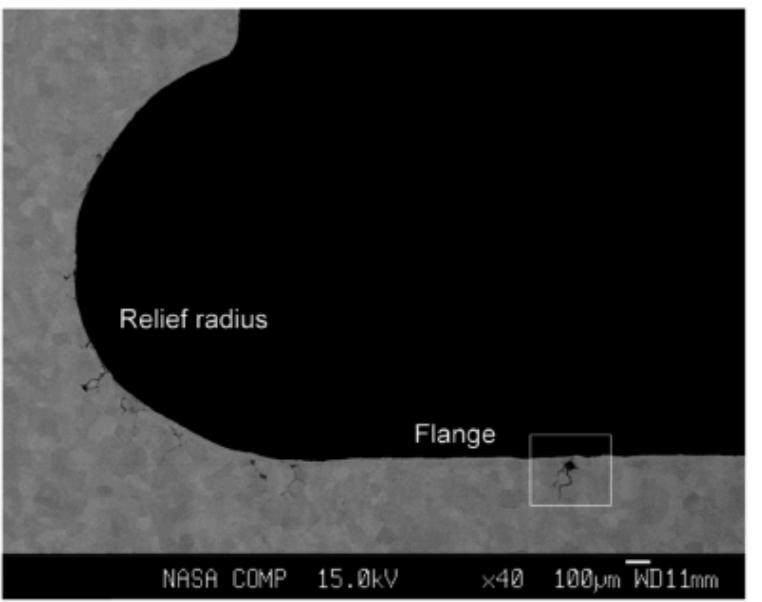

- $\quad$ Projected crack depth (a) and ligament distance (a') more critical to measure than actual crack depth.

R. A. MacKay, S. W. Smith, S. R. Shah, R. S. Piascik, "Reaction control system thruster cracking consultation: NASA Engineering and Safety Center (NESC) Materials Super Problem Resolution Team (SPRT) Findings, NASA/TP_-2005-214053.

2. B. Wincheski, "Eddy current techniques for nondestructive evaluation of complex materials and structures", NASA-Industry Partnership Workshop, Nondestructive Evaluation Sciences, June 20, 2006. Web site: www.industrynasapartnership.com/NASApresentations/Wincheski Eddy Current.pdf. 


\section{Background - Thruster Problem Model}

\section{Key Design Parameters:}
A. Part Geometry
Thruster (cut-away, use simplified geometry)
B. Part Material Properties
Niobium alloy (Grain noise, Roughness of cavity)
C. Crack Parameters
Crack length
$0.000 "-0.175 "$ (in terms of projected crack depth)
Desired detection range $0.020 "-0.060 "$ (in terms of remaining material)
Crack width open / intergranular cracks
Crack orientation (angle)
$30^{\circ}-60^{\circ}$ (between hole)

\section{Objectives for Case Study Problem:}

1. Apply Models to Optimize Inspection Design

Probe number:

Probe orientation:

Frequency (multiple):

Probe (coil) dimensions: single or differential probes (location) three different axial orientations with cavity

$1.0 \mathrm{kHz}-50 \mathrm{kHz}$

(constrained by hole diameter)

2. Explore Model-assisted POD Evaluation for NDE Technique Validation 


\section{Eddy Current NDE Modeling}

- Formulation

- Maxwell's Equations $\nabla \times \vec{E}=-j \omega \vec{B}$

$\nabla \times \vec{H}=j \omega \vec{D}+J^{(e)}$

$\nabla \cdot \vec{B}=0$

$\nabla \cdot \vec{D}=\rho$

- Magnetic Vector Potential Definition

$$
\nabla \times \vec{A}=\vec{B}
$$

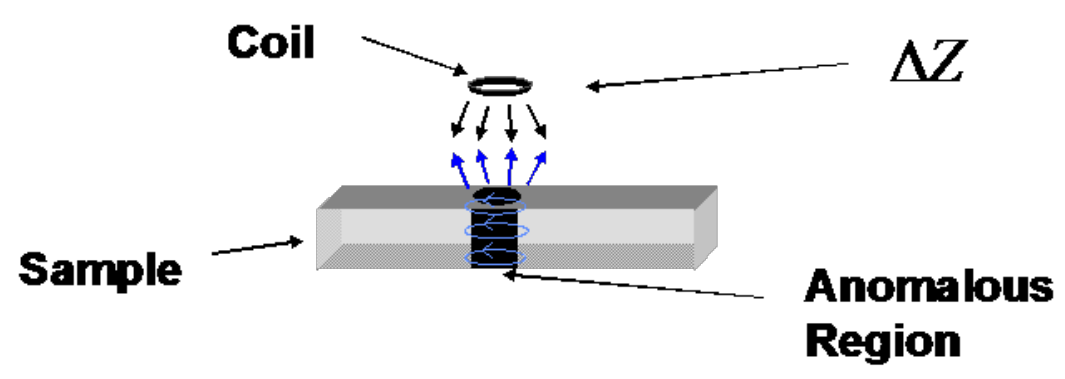

$=$ electric field

$=$ magnetic field

$=$ electric displacement

= charge density

$\vec{A}=$ magnetic vector potential

$\vec{J}_{0}=$ applied current density

$\mu=$ magnetic permeability

$\sigma=$ electrical conductivity

- Isotropic, Linear, Inhomogeneous Medium

$$
\nabla^{2} \vec{A}=\mu \vec{J}_{0}+\mu \sigma \frac{\partial \vec{A}}{\partial t}+\mu \nabla(1 / \mu) \times(\nabla \times \vec{A})
$$

- Coil impedance calculation $\quad Z=\frac{j \omega \int A \cdot d s}{I}$ 


\section{Numerical Methods}

\section{Methods:}

- Analytical - TREE (truncated region eignefunction expansion)

- Finite Difference Method (FDM)

- Finite Element Method (FEM) [OPERA-3D, COMSOL]

- Boundary Integral Equation Method (BIEM) [ECSIM]

- Volume Integral Method (VIM)

[VIC-3D]

- Meshless Methods

Advantages:

- Finite Element Method (Opera 3D)

- Efficient discretization of awkward geometries

- Calculations available anywhere within solution domain

- Volume Integral Method (VIC-3D)

- Only region of the scatterer need be discretized

- Fast 


\section{Thruster Problem Model}

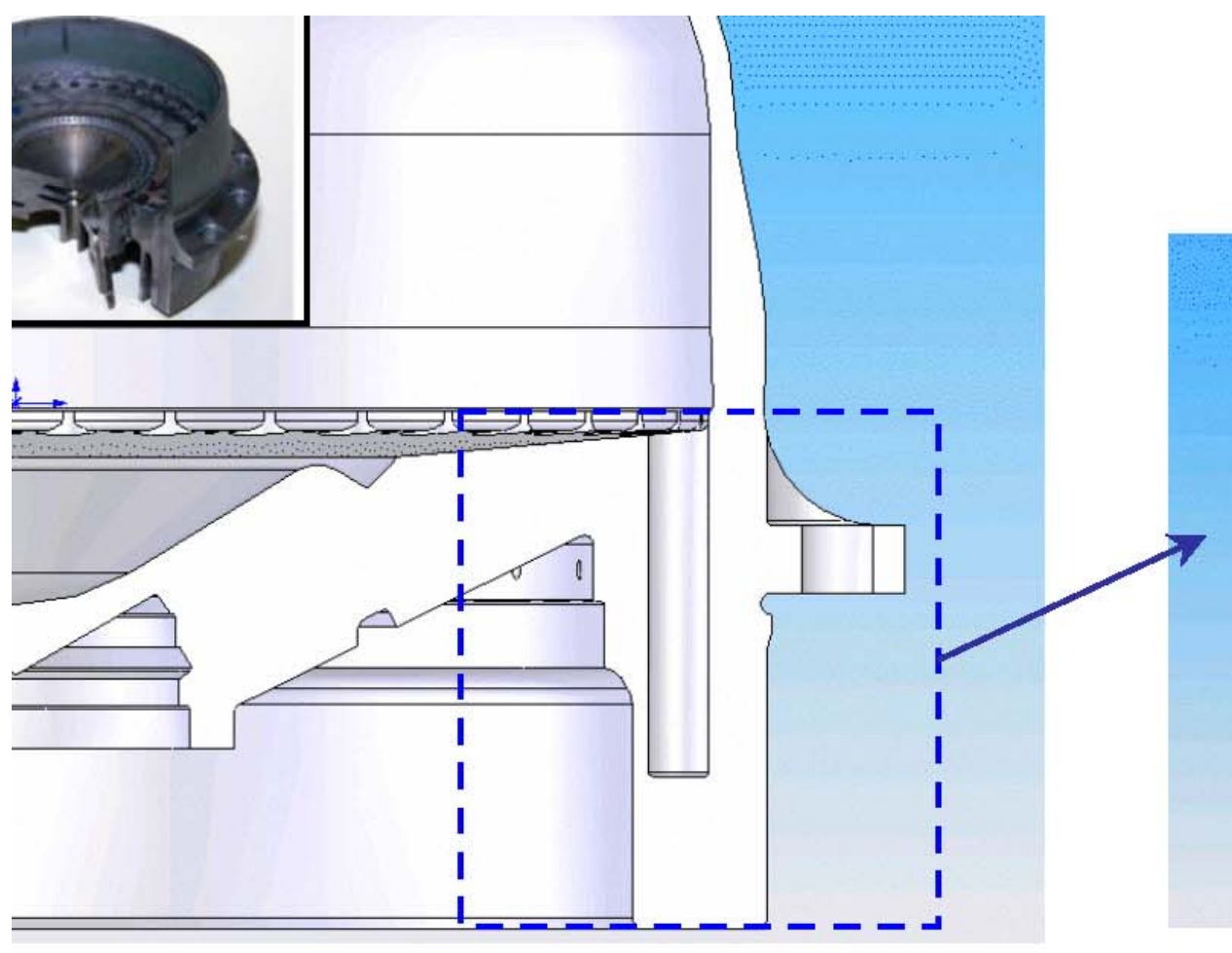

Thruster Cut-away

Simplified geometry for thruster model 


\section{Model - FEM (Opera-3D)}

\section{Thruster Simulations in Opera-3D:}

- 'Simplified geometry' with single coil constructed in Opera-3D

- Modeling options: user interface, script language, or CAD file Original CAD model did not mesh well (result in poor solution)

Converted CAD model into a script input file
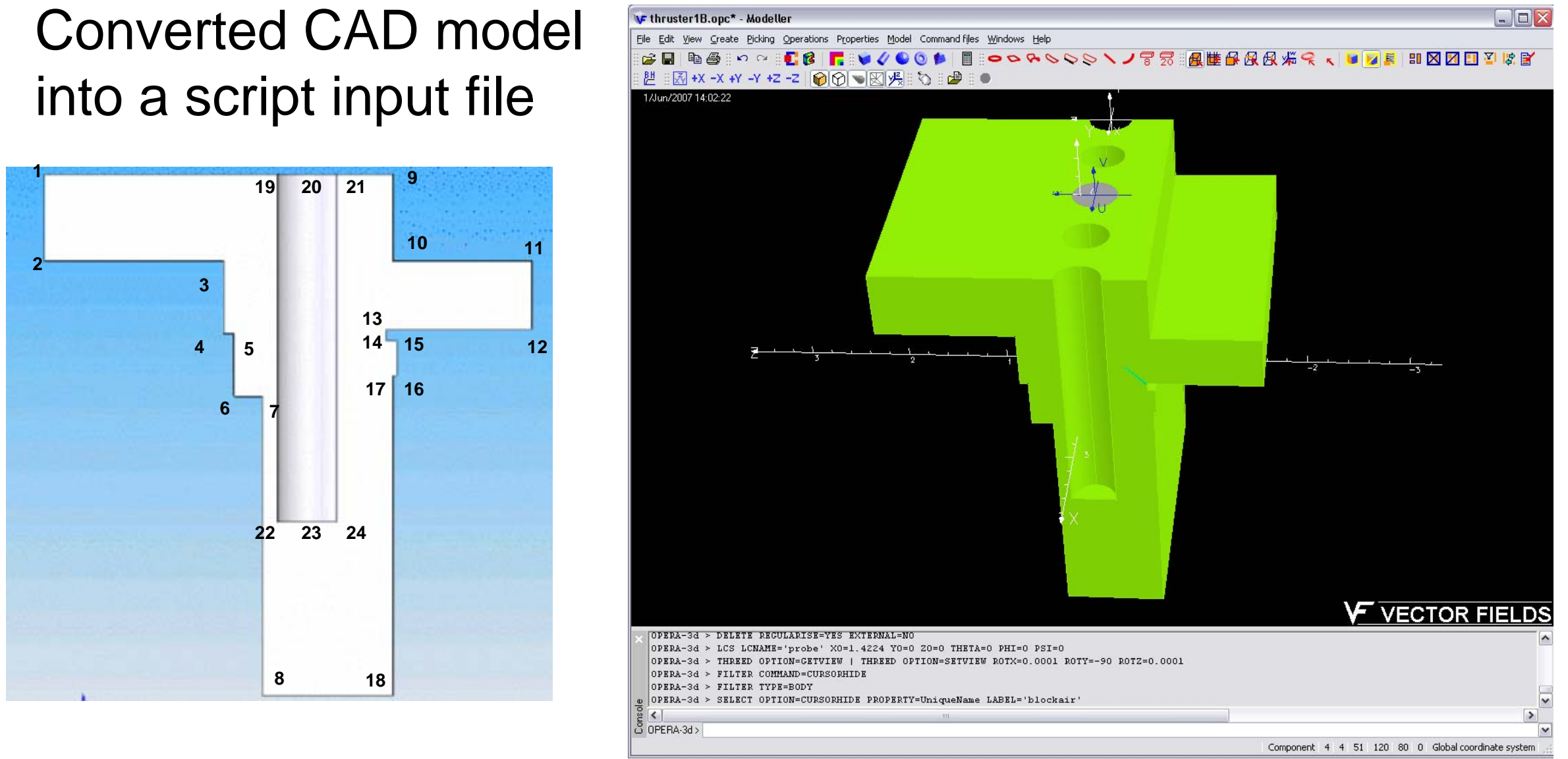


\section{Model - FEM (Opera-3D)}

\section{Thruster Simulations in Opera-3D:}

- Script file also required for running parametric studies

- crack length

- probe location

- probe orientation

- Impedance from dissipated energy by the conductor $(P)$ and stored energy (W) in domain

$Z=R+j \omega L$

$R=\frac{P}{I^{2}} \quad L=\frac{2 W}{I^{2}}$

$P=\int J^{e} E^{*} d \Gamma^{\prime} \quad W=\frac{1}{2} \int H B^{*} d \Gamma$

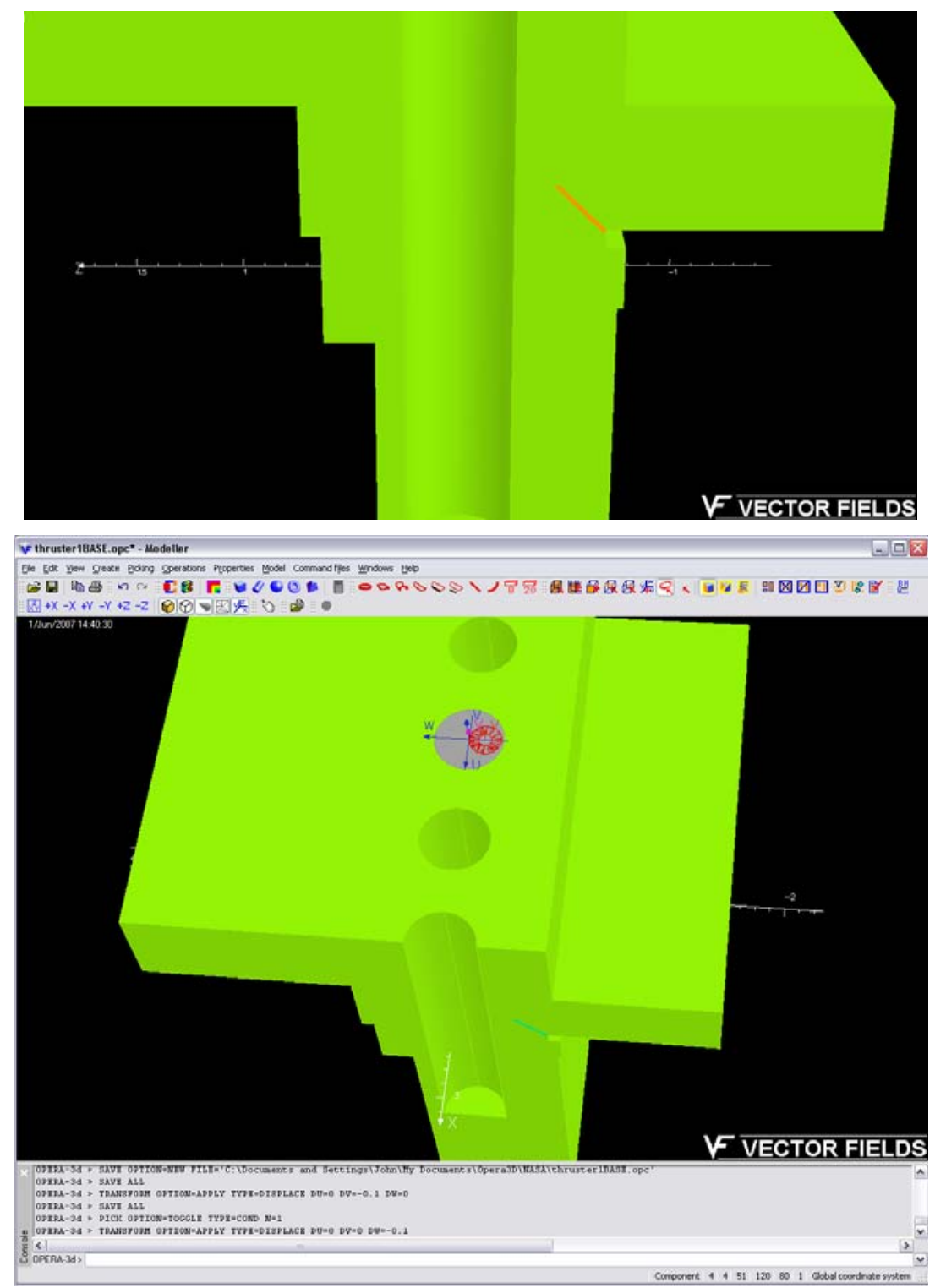




\section{Results - FEM (Opera-3D)}

\section{Thruster Simulation Results in Opera-3D :}

Absolute FEM results

- Irregular tetrahedral mesh produces significant variation for probe at varying locations

- Trends observed in response assoc. with part geometry

- Increasing mesh density significantly increases solution time

no flaw - FEM simulation - tang(2) coil

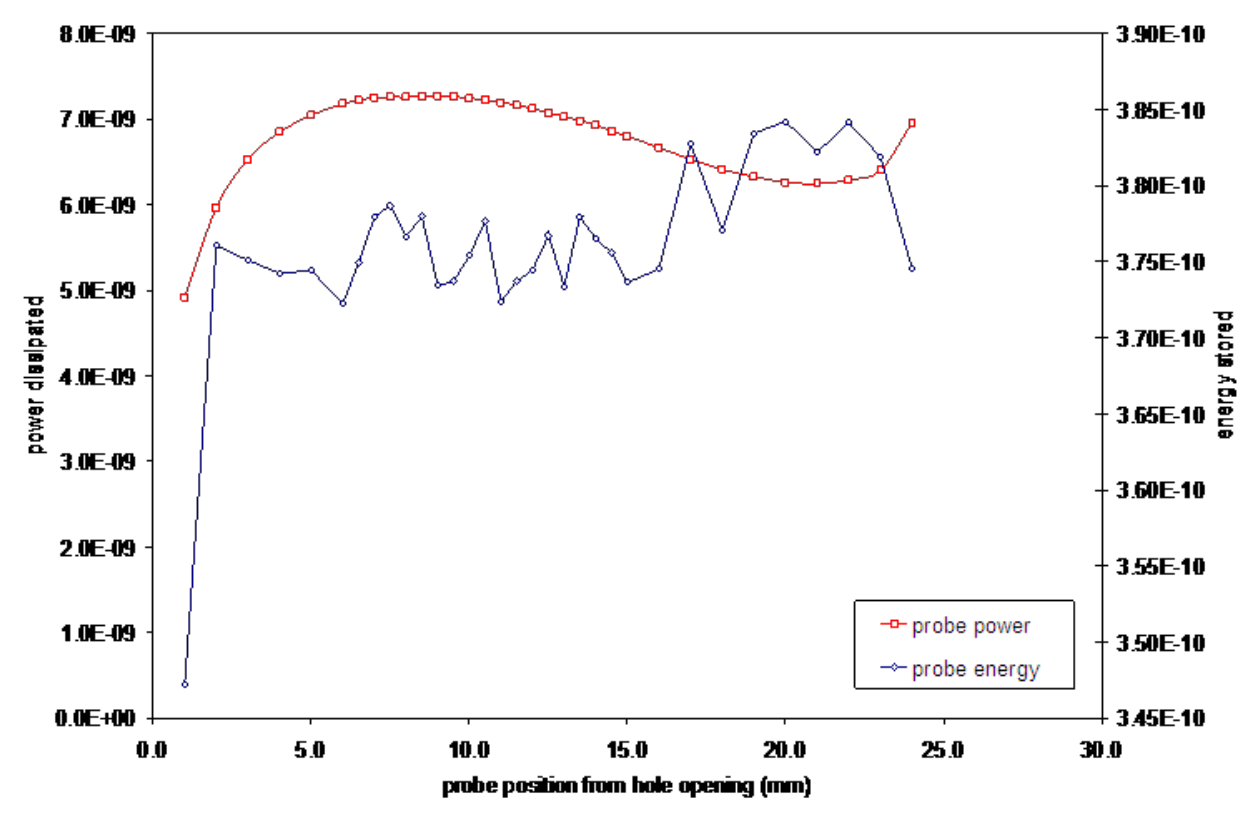

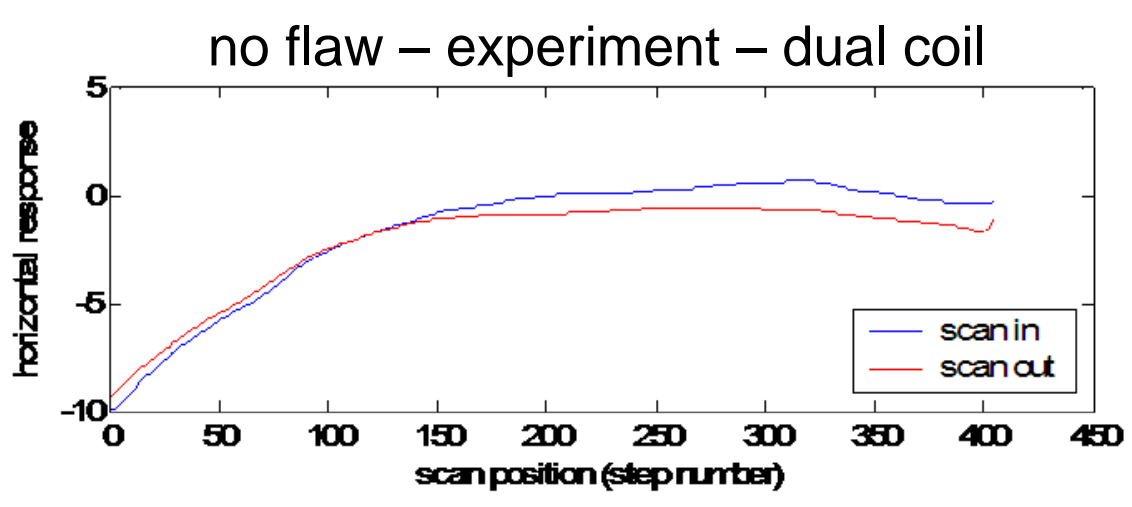

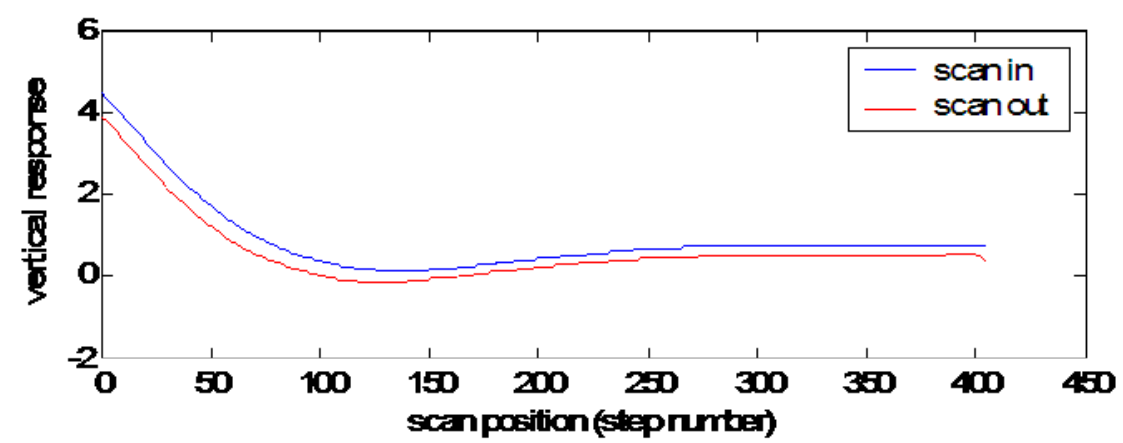




\section{Results - FEM (Opera-3D)}

\section{Thruster Simulation Results in Opera-3D :}

\section{Differential FEM results}

- Mesh variation error can be simply addressed by solving for both 'no flaw' and 'with flaw' conditions and taking difference

- Localized response

- Simulation time (two conditions * 26 probe locations): $\sim 36 \mathrm{hrs}$
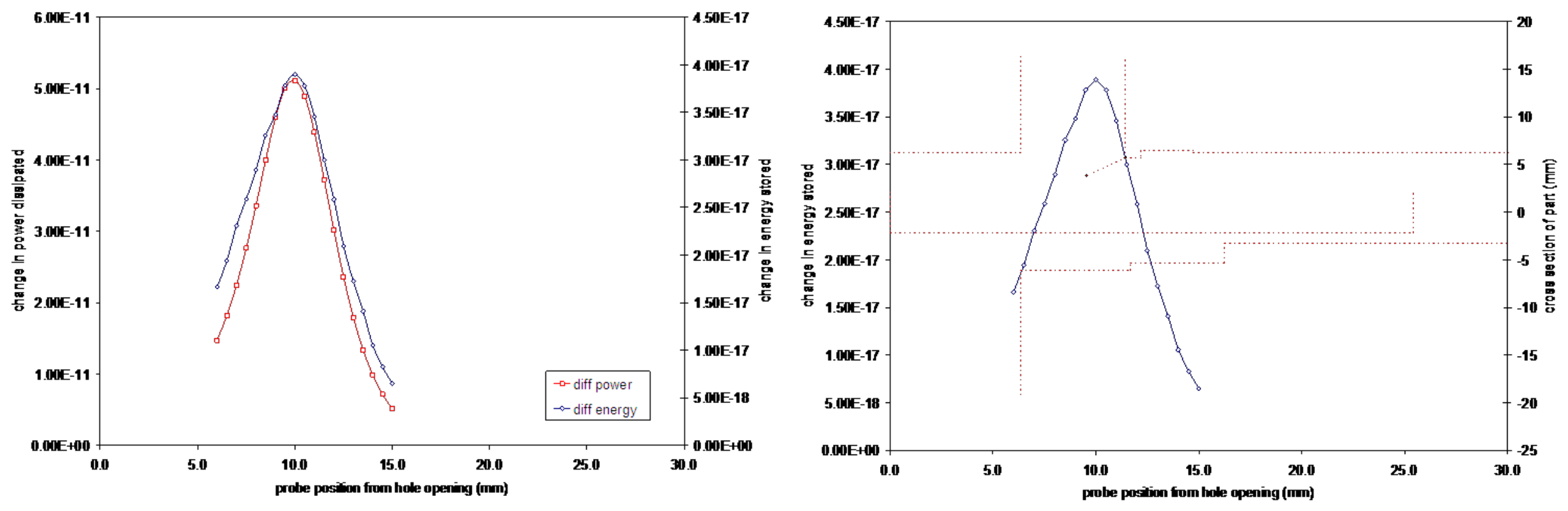

4. D. C. Carpenter, 'Use of the finite element method in simulation and visualization of electromagnetic nondestructive testing applications,' Materials Evaluation, Vol. 58, No.7, pp. 877-881, 2000 


\section{Comparison with Experiment}

\section{Experimental Results:}

Orthogonal probe $12 \mathrm{kHz}$



\section{Volume Integral Method Formulation}

- Start with Maxwell's equations

$$
\begin{aligned}
& \nabla \times \mathbf{E}=-j \omega \mathbf{B} \\
& \nabla \times \mathbf{H}=-j \omega \mathbf{D}+\mathbf{J}^{(e)}
\end{aligned}
$$

- Restrict anomalous (flaw) regions to a layer (can address layers using spatial decomposition algorithms)

- Form system of volume integral equation's using Galerkin's method

- Solve system of equations to evaluate scattering (anomalous) currents in the flaw region (Given incident field due to excitation coil)

- Due to the Toplitz-Hankel structure of the equations, a 3D-FFT conjugate gradient algorithm can be used to solve large problems. 


\section{Simulated Response to Structure}

\section{Noise Distribution:}

- Variation due to structure Model Trends Match Experiment:

- $12 \mathrm{KHz}$ data

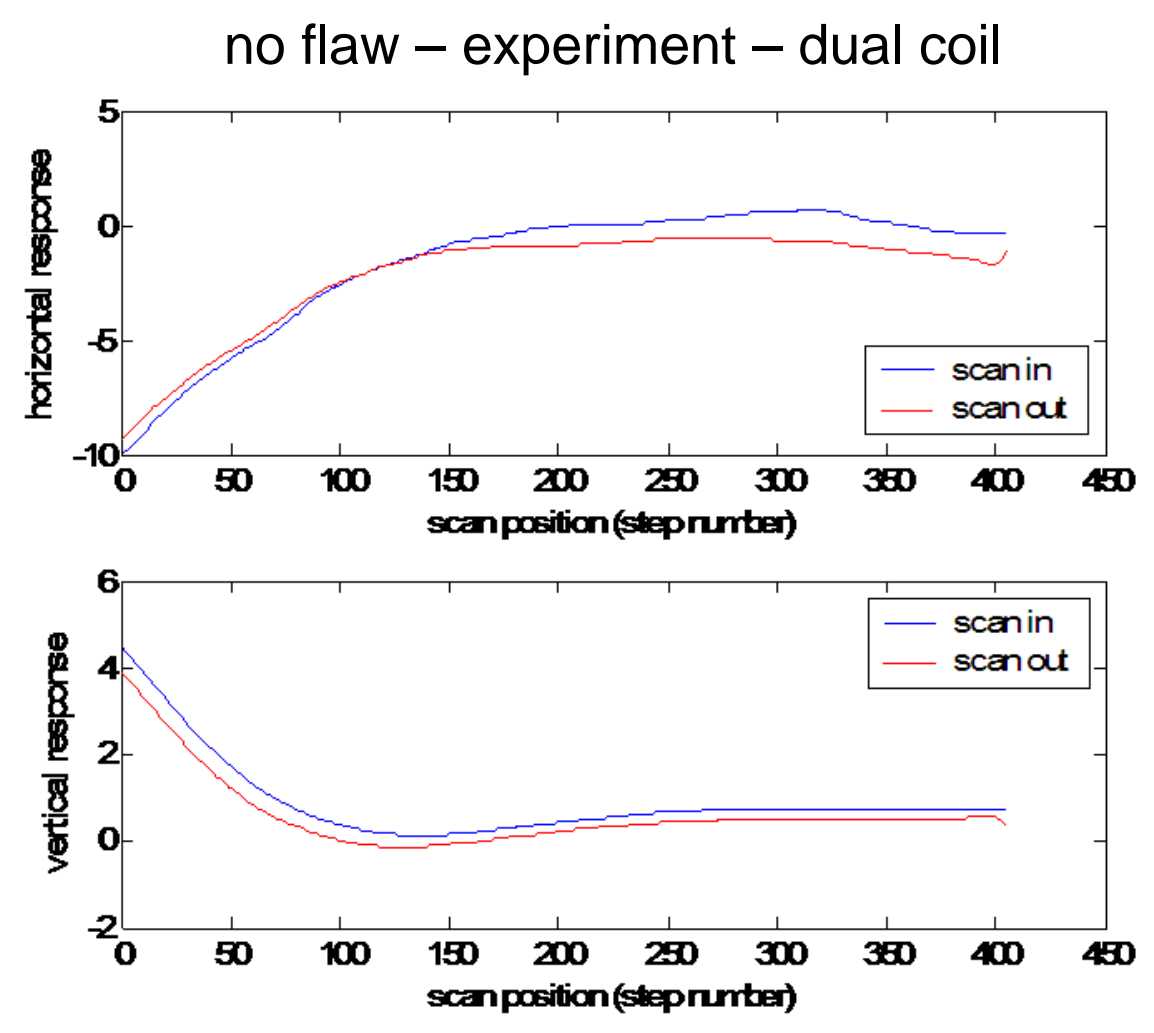



no flaw - model - tangential probe (2)

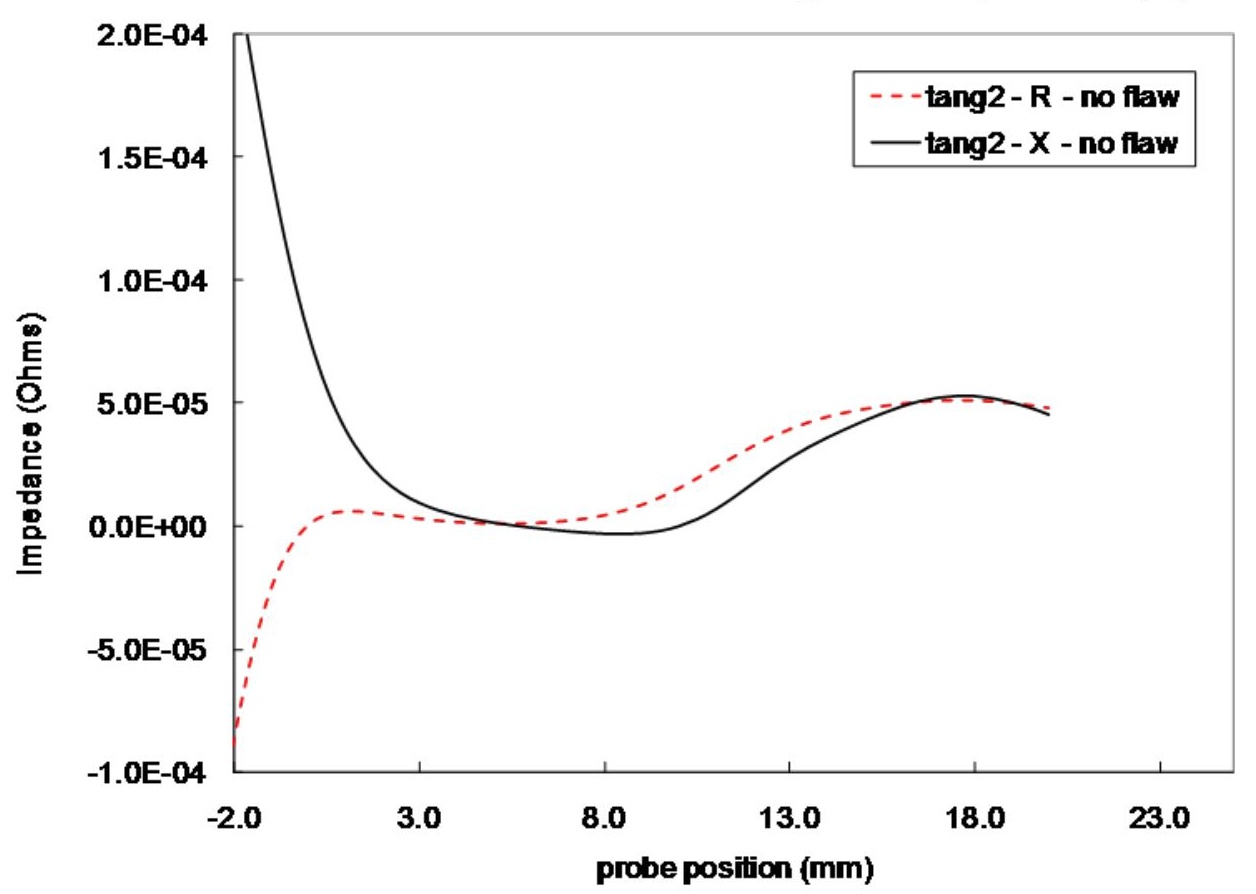




\section{Response to Crack}

\section{Crack Signal Distribution:}

- Function of crack size

- Compare experiment and simulation



with flaw (0.5 $\mathrm{mm}$ remaining wall thickness)

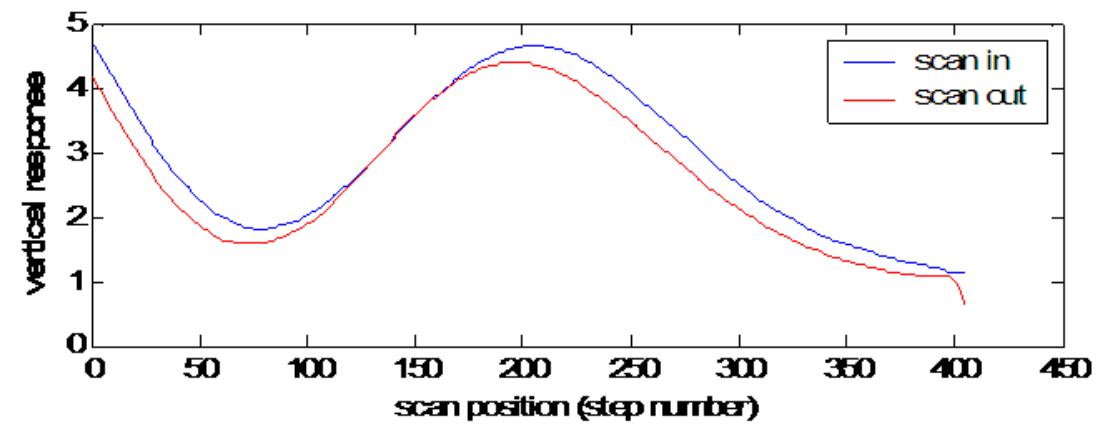

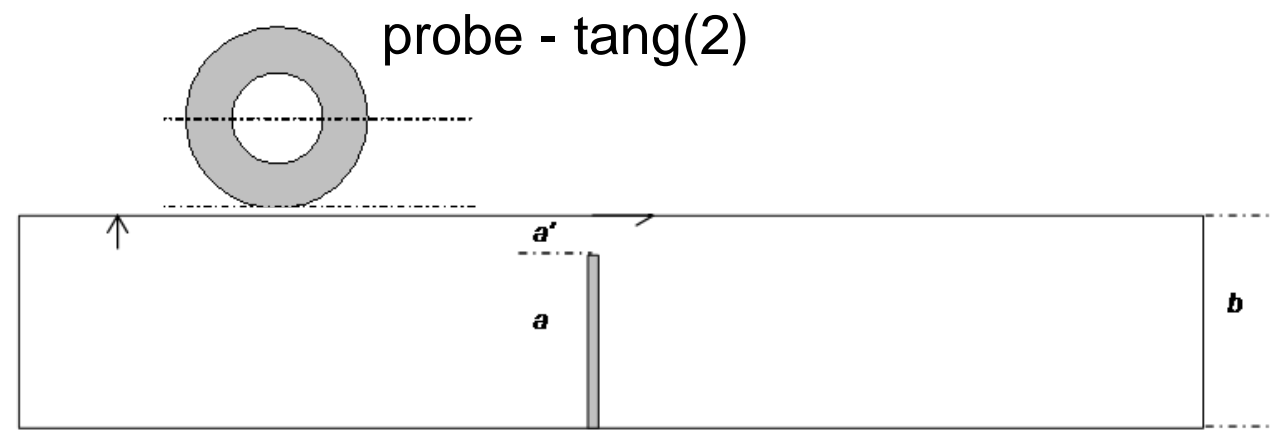

with flaw (0.5 $\mathrm{mm}$ remaining thickness)

- model - tangential probe (2)

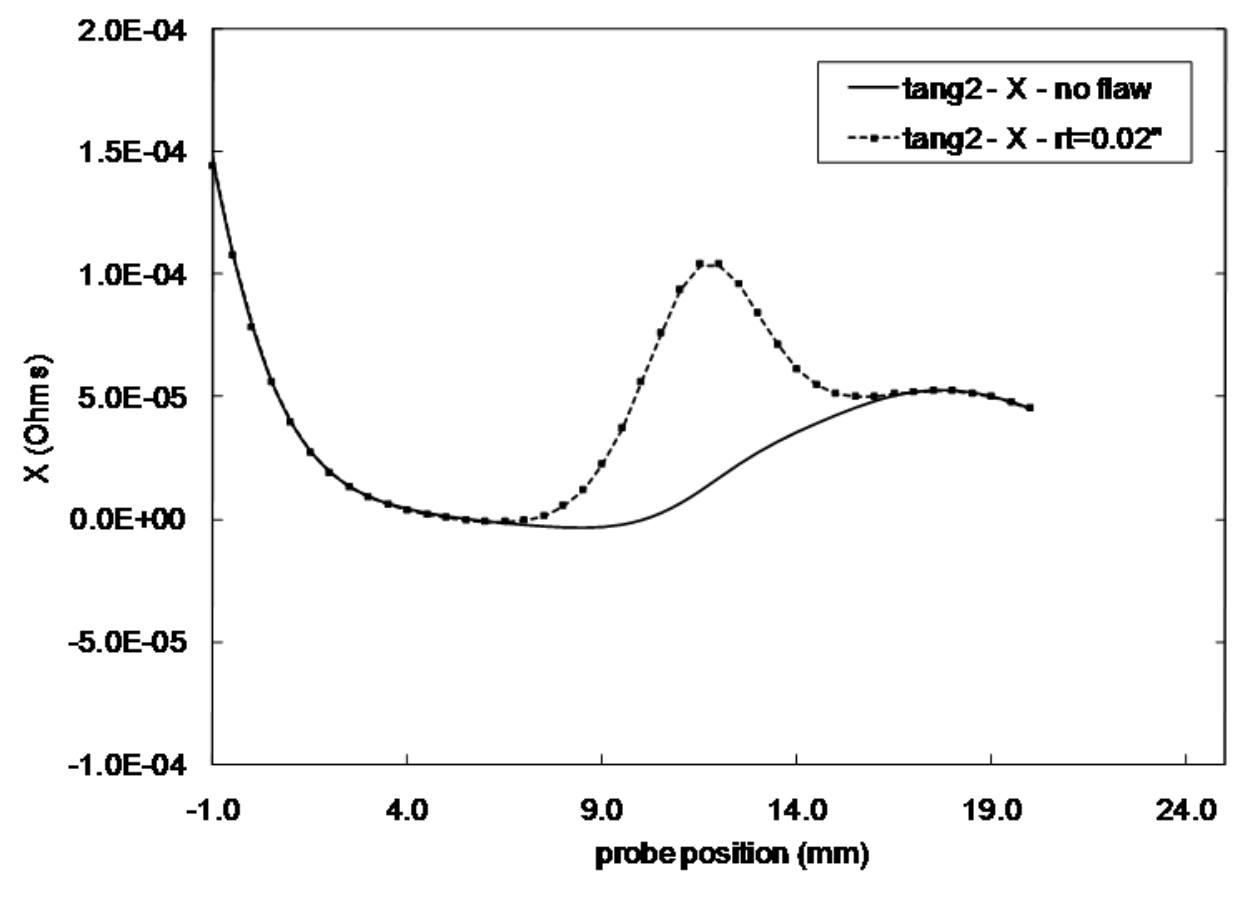




\section{Sensitivity to Crack}

\section{Crack Signal Distribution:}

- Function of crack size

- Compare experiment and simulation

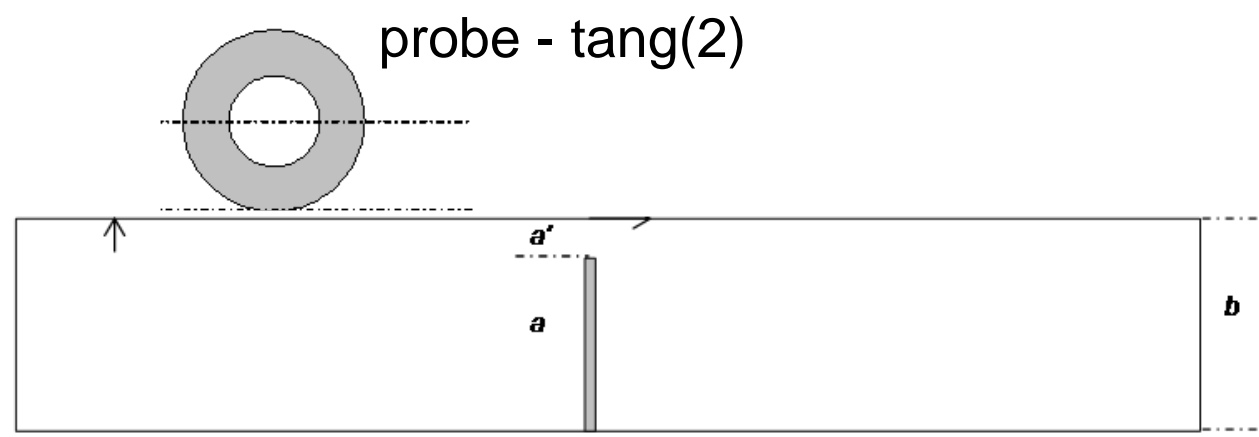

vary remaining wall thickness experiments - dual coil 「61

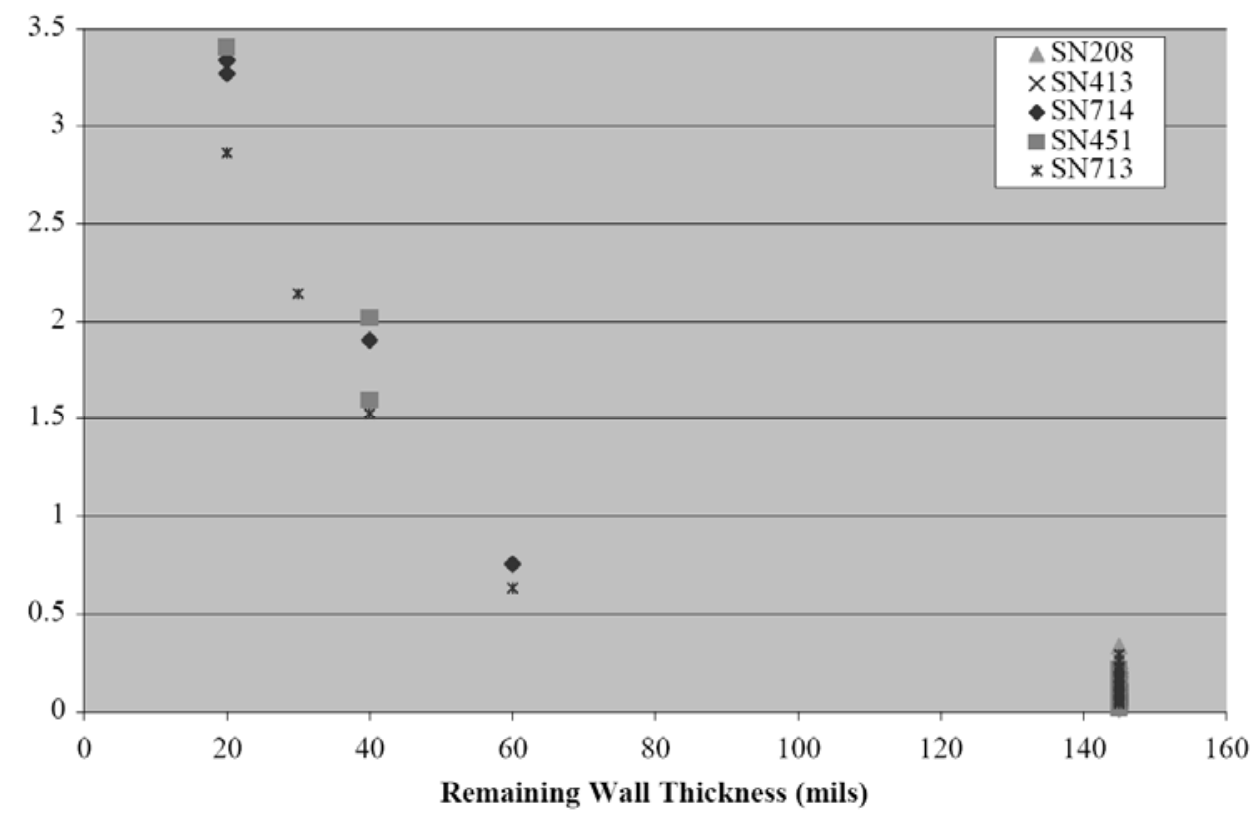

with notch - model - tangential probe (2)



6. B. Wincheski, J. Simpson, A. Koshti, "Development of Eddy Current Techniques for the

Detection of Cracking In Space Shuttle Primary Reaction Control Thrusters" NASA/TP-2007-21487. 


\section{Sensitivity to Crack}

\section{Crack Signal Distribution:}

- Function of crack size

- Vary orientation and remaining thickness

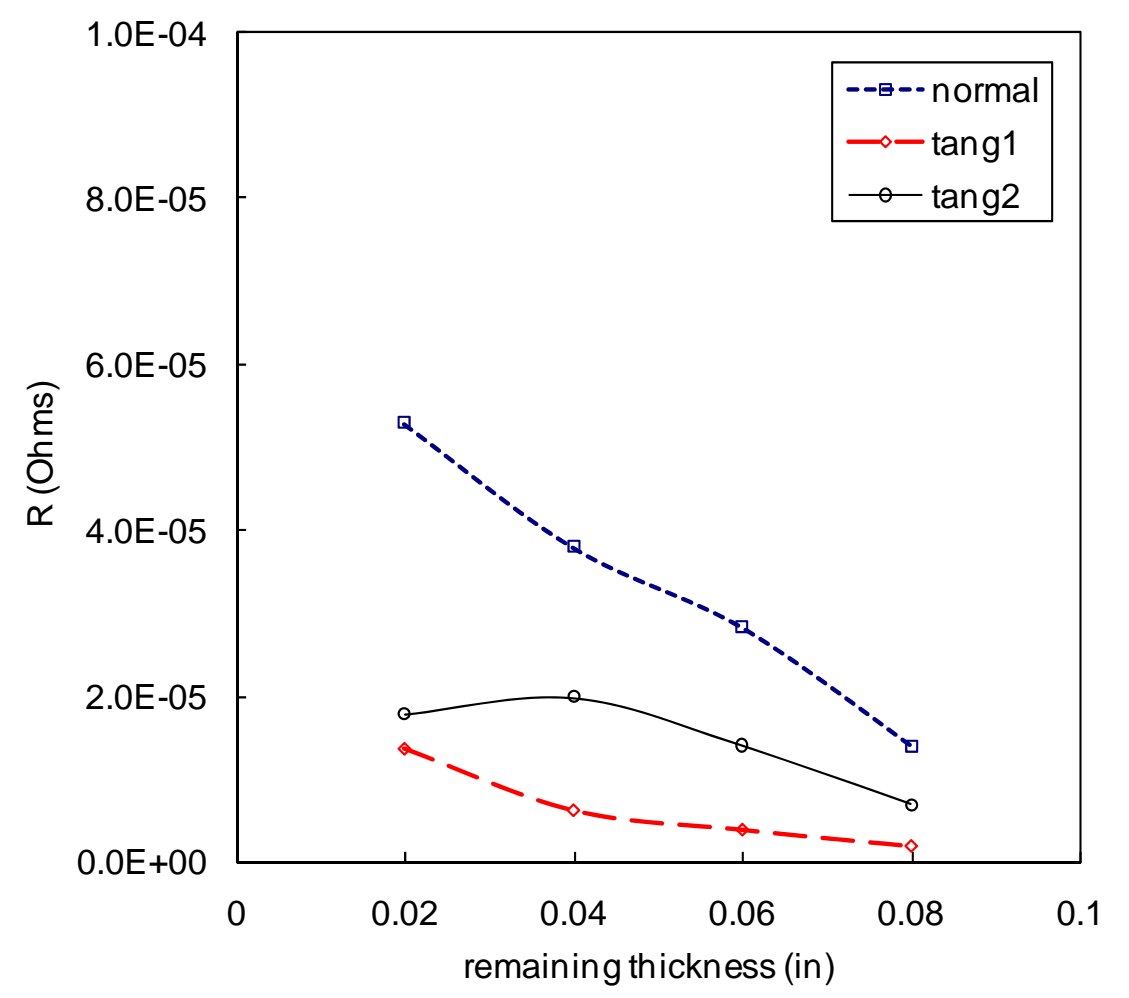

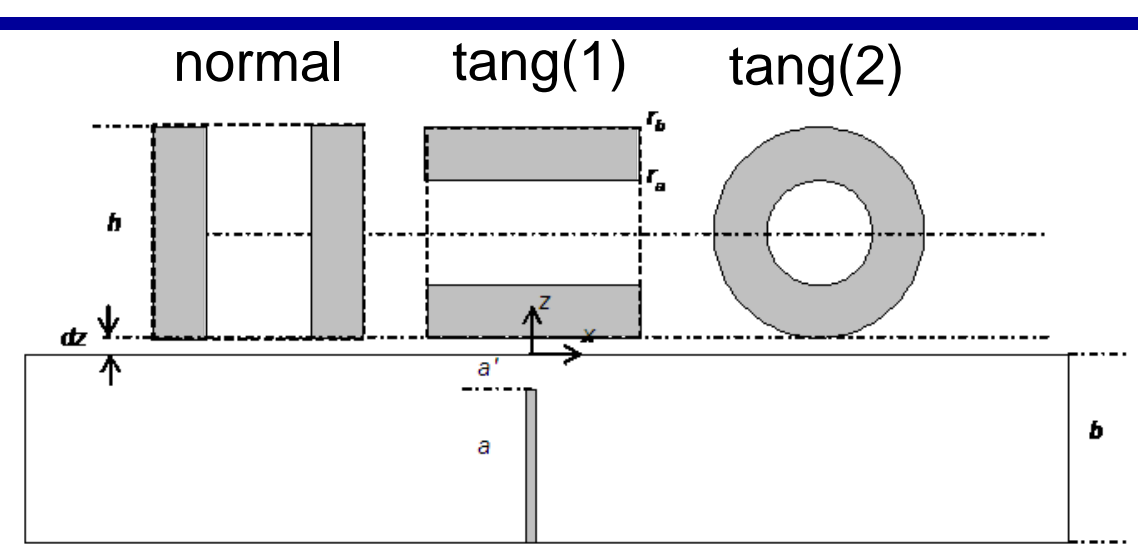






\section{Sensitivity Analysis - VIC-3D}

- Evaluate measurement sensitivity as function of frequency and notch length

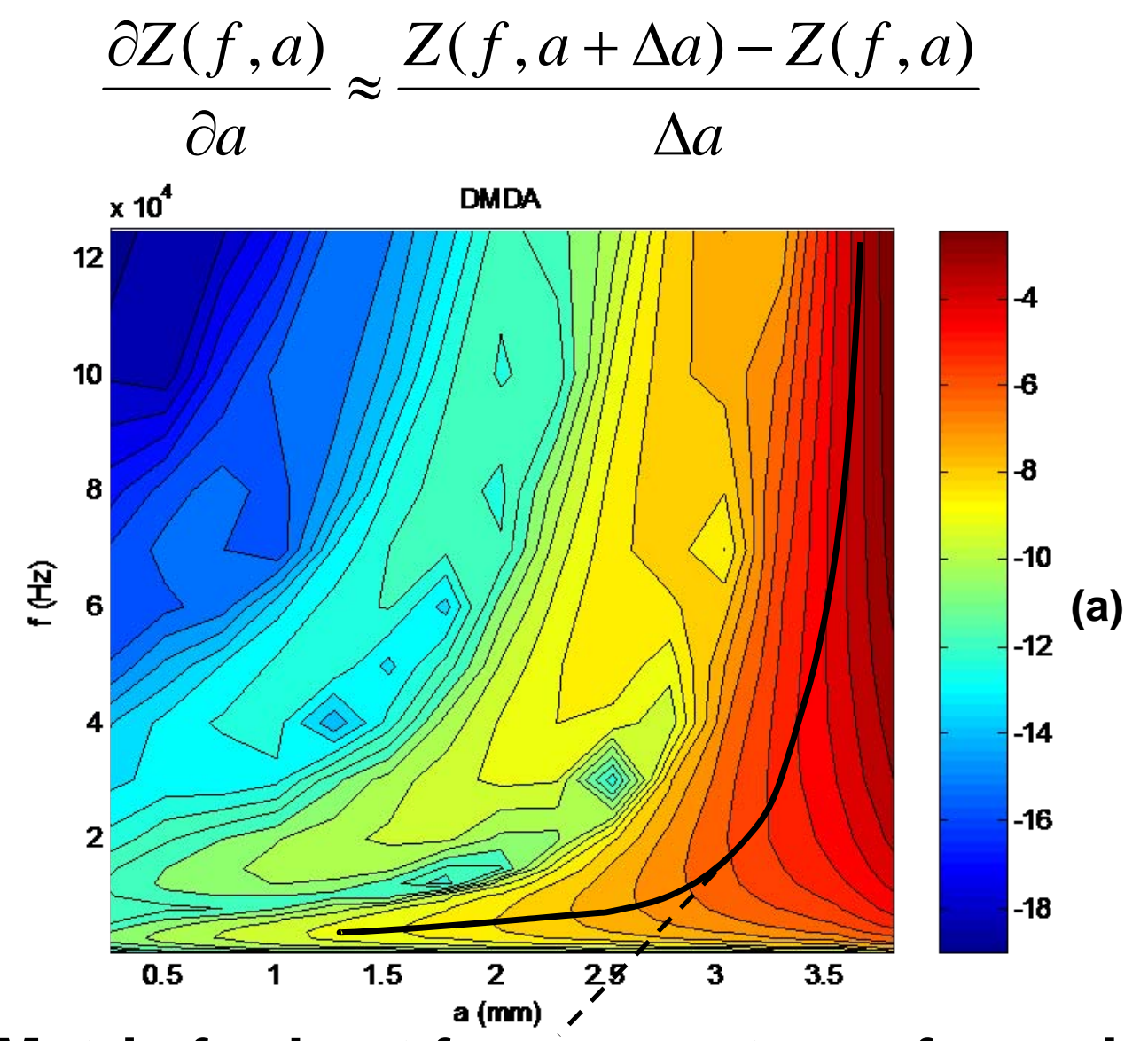

Metric for best frequency to perform sizing


(c) 


\section{Making Quantitative Comparisons}

\section{- Prior Work - FEM of eddy current densitv (Wincheski et al. 2007)}

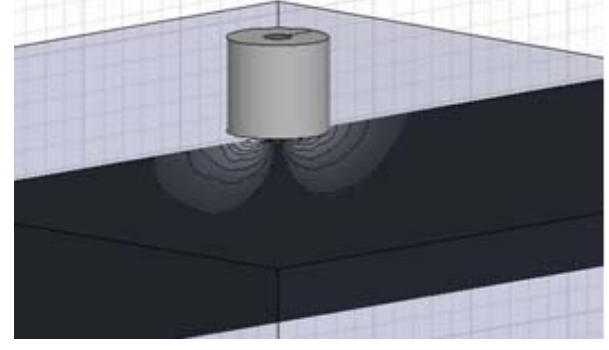

normal

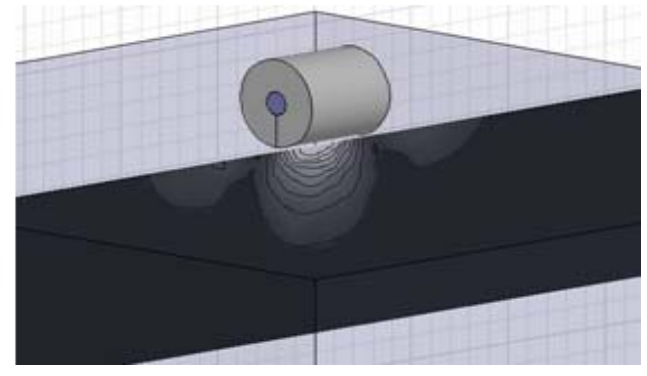

tangential

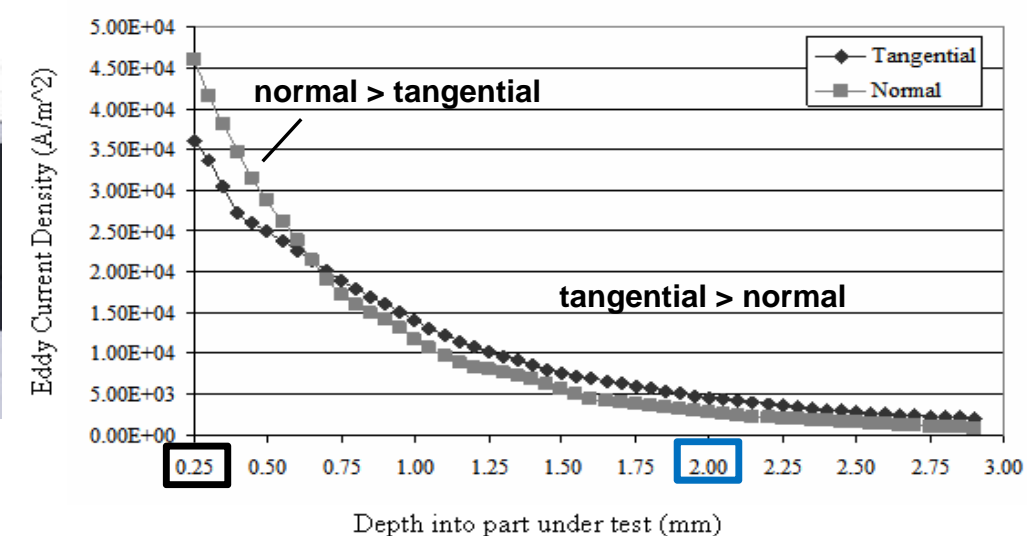

Depth into part under test $(\mathrm{mm})$

- VIC-3D: EC measurement is due to 1) magnitude of eddy current density in flaw region and 2) measurement model of disturbed currents
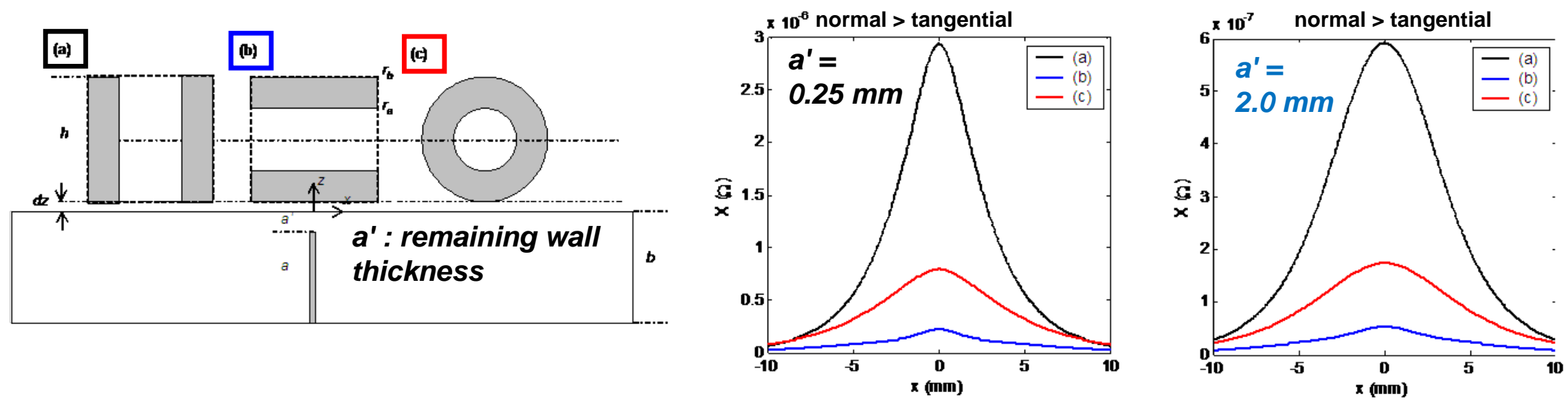

- To make an accurate comparison between designs, there is need to also include all associated variances (noise factors) in measurements 


\section{Model-assisted POD Protocol (Thompson et al)}

1. Identify the scope of the POD study

2. Identify factors that control signal and noise

3. Evaluate quality of physics-based models

4. Acquire / develop / validate simulation tools

5. Acquire input parameters / parameter distributions

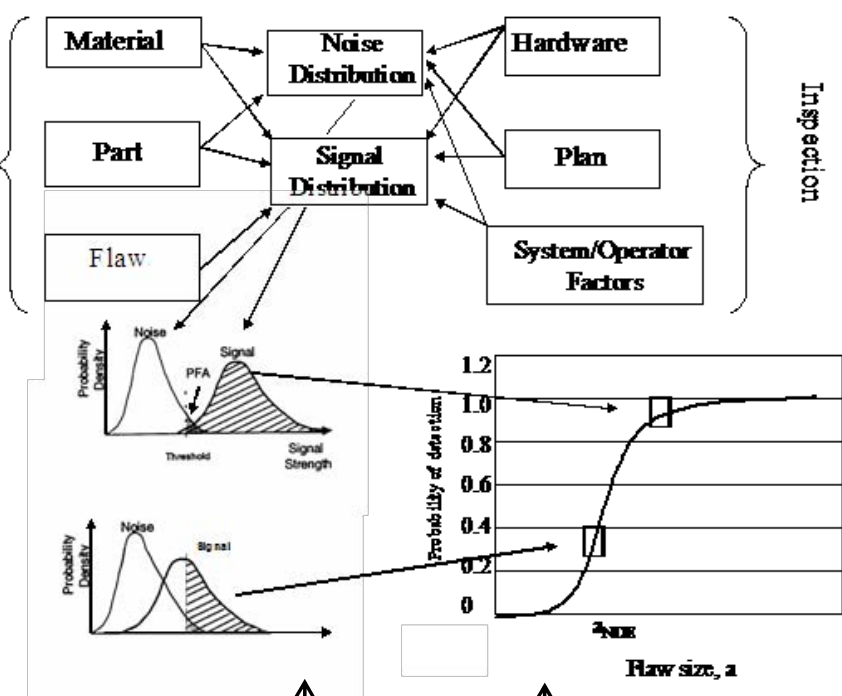

6. Conduct flaw signal distribution simulations and noise signal distribution simulations

7. Acquire remaining information on factors empirically

8. Acquire marginal information on independent factors and covariance information on dependent factors

9. Evaluate full signal and noise distributions [ f(crack length)]

10. Compute POD with Probability of False Call (POFC) [Monte Carlo] 


\section{Model-assisted POD Protocol}

Noise Distribution:

- Variation due to measurement (reference, self calibration)

- Variation due to structure

- Variation due to probe

- orientation (angular)

- liftoff from surface

Crack Signal Distribution:

- Function of crack size (mean)

- Variation due to crack geometry (+ probe)

- initiation site

- crack orientation (angle)

+ Include noise distribution
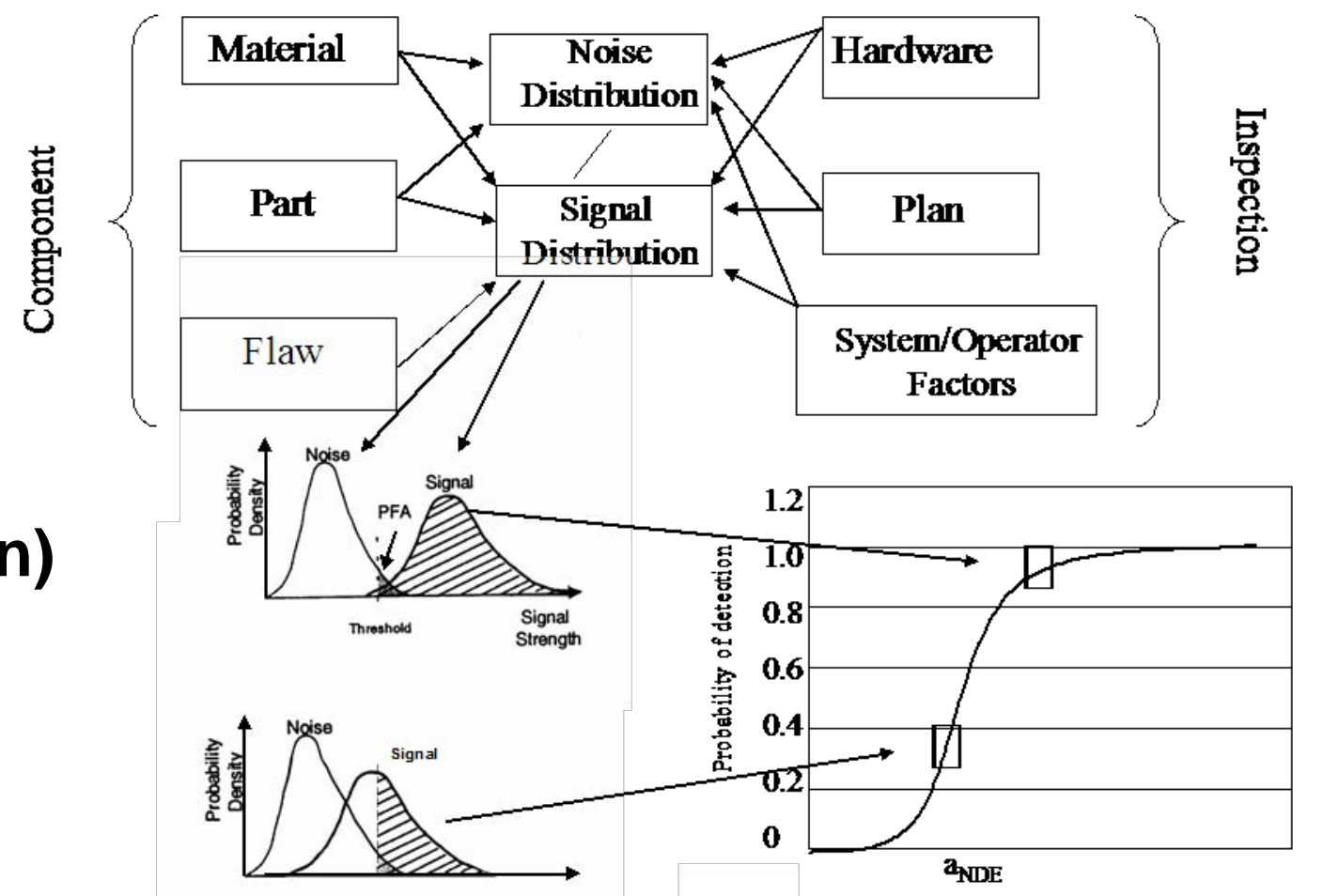

Flaw size, a

(Thompson et al) 


\section{Sensitivity to Structure}

\section{Noise Distribution: Variation due to structure}

\section{Use Approximate Model - VIC-3D simulations (12 kHz)}

- $\quad$ Split model into two parts (each half of structure)

- Evaluate sensitivity of probe orientation to structure
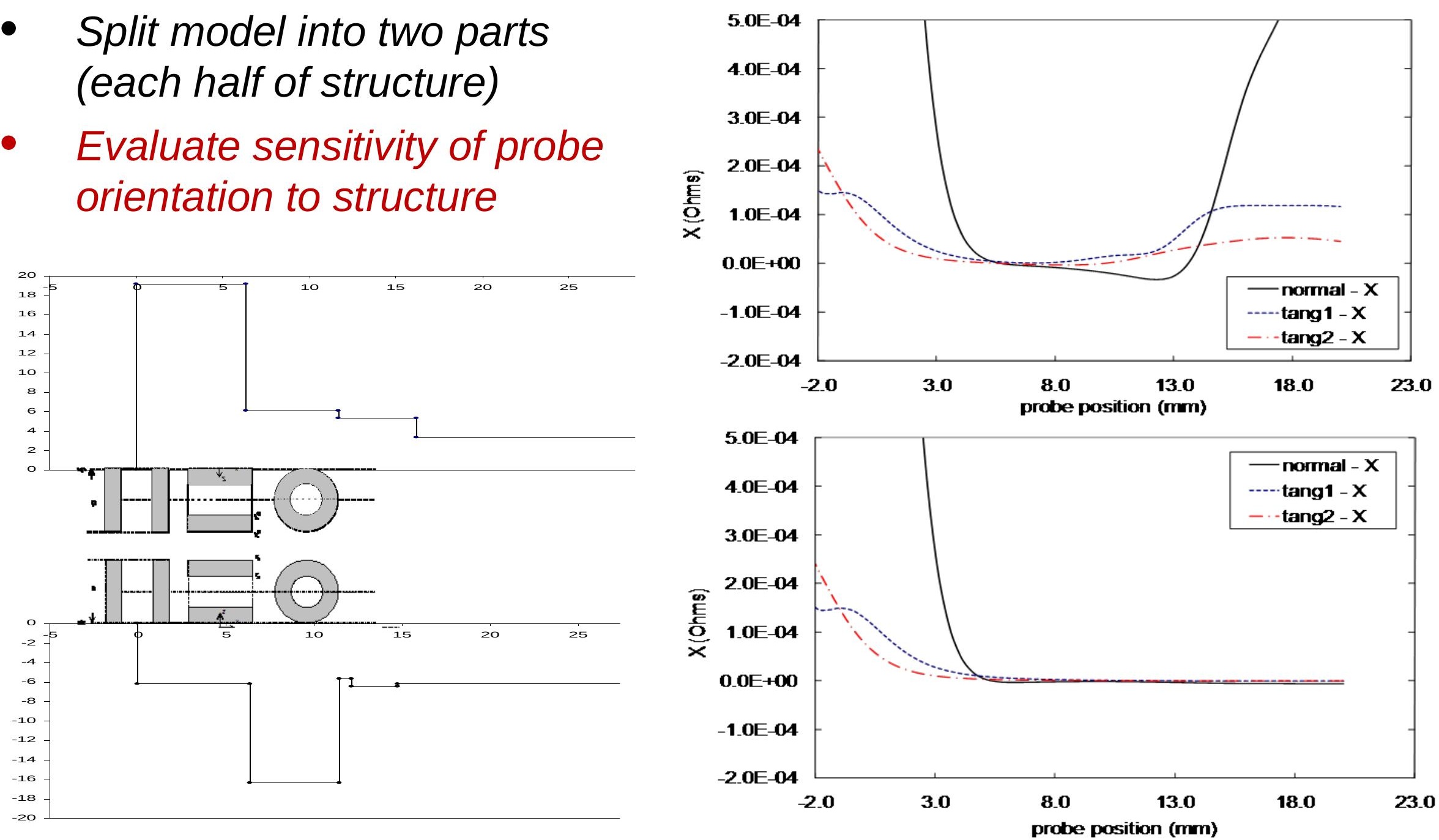


\section{Summary and Future Work}

\section{Summary:}

- Two numerical methods, FEM and VIM, were used to simulate eddy current NDE for cracks in a complex thruster geometry

- Model demonstrated trends observed in experimental studies

- Sensitivity studies performed to determine the ideal probe orientations and frequencies for varying crack lengths.

- To make an accurate comparison between designs using simulation:

- Need accurate measurement models

- Must include all critical variances in measurements

- MAPOD study outlined with preliminary design results

Future Work:

- Complete full model-assisted POD (MAPOD) evaluation

- Explore hybrid models to efficiently solve for multiscale geometries

- Investigate optimum designs for improving detection of deep cracks 


\section{Acknowledgements}

- Bill Winfree and Bill Prosser, NASA Langley

- Victor Technologies (VIC-3D ${ }^{\circledR}$ )

- Vector Fields (Opera-3D ${ }^{\circledR}$ ) 